\title{
High-Resolution Climate Simulations Using GFDL HiRAM with a Stretched Global Grid $\mathscr{a}$
}

\author{
LUCAS M. HARRIS AND SHIAN-JIANN LIN \\ NOAA/Geophysical Fluid Dynamics Laboratory, Princeton University, Princeton, New Jersey \\ CHIAYING TU \\ Research Center for Environmental Changes, Academia Sinica, Taipei, Taiwan
}

(Manuscript received 2 June 2015, in final form 15 March 2016)

\begin{abstract}
An analytic Schmidt transformation is used to create locally refined global model grids capable of efficient climate simulation with gridcell widths as small as $10 \mathrm{~km}$ in the GFDL High-Resolution Atmosphere Model (HiRAM). This method of grid stretching produces a grid that varies very gradually into the region of enhanced resolution without changing the topology of the model grid and does not require radical changes to the solver. AMIP integrations were carried out with two grids stretched to 10-km minimum gridcell width: one centered over East Asia and the western Pacific warm pool, and the other over the continental United States. Robust improvements to orographic precipitation, the diurnal cycle of warm-season continental precipitation, and tropical cyclone maximum intensity were found in the region of enhanced resolution, compared to $25-\mathrm{km}$ uniform-resolution HiRAM. The variations in grid size were not found to create apparent grid artifacts, and in some measures the global-mean climate improved in the stretched-grid simulations. In the enhancedresolution regions, the number of tropical cyclones was reduced, but the fraction of storms reaching hurricane intensity increased, compared to a uniform-resolution simulation. This behavior was also found in a stretchedgrid perpetual-September aquaplanet simulation with $12-\mathrm{km}$ resolution over a part of the tropics. Furthermore, the stretched-grid aquaplanet simulation was also largely free of grid artifacts except for an artificial Walker-type circulation, and simulated an ITCZ in its unrefined region more resembling that of higherresolution aquaplanet simulations, implying that the unrefined region may also be improved in stretched-grid simulations. The improvements due to stretching are attributable to improved resolution as these stretchedgrid simulations were sparingly tuned.
\end{abstract}

\section{Introduction}

Locally high-resolution simulations are necessary for many applications in atmospheric science where smallscale features need to be resolved, such as hurricane forecasts or regional climate simulation, when a globally uniform high-resolution simulation would be impractical. A limited-area model is typically used for these

Supplemental information related to this paper is available at the Journals Online website: http://dx.doi.org/10.1175/ JCLI-D-15-0389.s1.

Corresponding author address: Lucas Harris, NOAA/Geophysical Fluid Dynamics Laboratory, Princeton University, Forrestal Campus, 201 Forrestal Rd., Princeton, NJ 08540.

E-mail: lucas.harris@noaa.gov purposes, but such models require boundary conditions supplied from a global model with potentially very different model design, possibly creating inconsistencies and errors at the model boundary. Further, limited-area models are unable to interact with the larger scales. A locally refined global grid avoids these problems. One form of local refinement is to stretch or deform the global grid (Courtier and Geleyn 1988; McGregor 2005; Fox-Rabinovitz et al. 2006; Tomita 2008; McGregor and Dix 2008; Uchida et al. 2016), such as by the analytic transformation of Schmidt (1977). A stretched grid is topologically identical to the original grid and so is conceptually simpler than a nested grid (Harris and Lin 2013, 2014, henceforth HL14) or an unstructured grid (cf. Thuburn et al. 2009; Rauscher et al. 2013) and does not require radical modification to the model formulation. The refinement in a stretched grid can also be much 
more gradual than the abrupt refinement of a nested grid, and thereby would be expected to cause fewer grid artifacts. However, stretching the grid causes a degradation of the resolution on the opposing side of the sphere, and the choices of time step and physical parameterization settings are the same globally regardless of resolution, scale-aware parameterizations excepted (cf. Walko et al. 2010). Summaries of earlier work on variable-resolution climate modeling are presented by Satoh et al. (2014), Zarzycki et al. (2015), Déqué and Piedelievre (1995), McGregor (2015), and references therein.

A version of the Geophysical Fluid Dynamics Laboratory (GFDL) High-Resolution Atmosphere Model (HiRAM; Zhao et al. 2009, henceforth Z09) has been developed using a Schmidt (1977) transformation to deform the (quasi-) uniform resolution cubed-sphere grid into a grid with one region of enhanced resolution, which smoothly relaxes to coarser resolution on the opposite side of the domain. This deformed or "stretched" grid can then be used in HiRAM in the same manner as a uniform-resolution cubed-sphere grid, albeit with the need to take a shorter time step due to the reduced size of the smallest grid cells.

We describe two series of stretched-grid and (quasi-) uniform simulations to show the effects of locally enhanced resolution. The first series is a set of idealized aquaplanet simulations, forced by fixed September-like SSTs and equinoctial insolation, in which the gridcell width is about $12 \mathrm{~km}$ in the enhanced-resolution region. The simplified framework and hemispherically asymmetrical SST permit study of the effects of resolution on the character of tropical precipitation and intertropical convergence zone (ITCZ), and of tropical cyclones (TCs). The stretched grid's average precipitation and TC activity is compared to those in (quasi-) uniform resolution simulations using an unstretched grid to determine how well the stretched-grid model can reproduce the TC activity, as a function of resolution, in the uniform-resolution simulations.

The second set of simulations is a set of comprehensive climate simulations forced by observed SSTs following the Atmospheric Model Intercomparison Project (AMIP) protocol for the period 1981-2008, in which a uniform-resolution $25-\mathrm{km}$ simulation is compared against a pair of stretched-grid simulations of minimum gridcell width of about $10 \mathrm{~km}$ : one with the refined region centered on Taiwan and covering the western $\mathrm{Pa}-$ cific warm pool and East Asia, and the other with the refined region centered on Oklahoma City and covering the continental United States, and the tropical eastern Pacific and western North Atlantic. We will look at the effect of enhanced resolution on biases in tropical and orographic precipitation, the diurnal cycle of continental precipitation, and tropical cyclone frequency and intensity. We will also look at the effect of local refinement on global-scale circulations to determine if improvement due to grid refinement can reach beyond the refined region. A thorough evaluation of HiRAM has been presented in previous articles (Z09; Chen and Lin 2013; Xiang et al. 2015, HL14), although standard climate model evaluation metrics will be computed for the stretched-grid simulations.

Section 2 summarizes HiRAM's dynamical core and parameterizations and describes the Schmidt transformation to create the stretched grid. Section 3 describes the aquaplanet simulations and compares the ITCZ and TCs in a stretched-grid simulation against a series of uniform-resolution simulations of comparable resolutions to different regions of the stretched-grid simulation. Section 4 describes the 1981-2008 comprehensive climate simulations and compares the uniformresolution and the two stretched-grid simulations against observations of precipitation and TC activity.

\section{Model formulation}

GFDL HiRAM has been designed for efficient highresolution simulations, with an emphasis on tropical precipitation and tropical cyclones (Z09; Chen and Lin 2011, 2013). Our HiRAM setup largely follows that in Chen and Lin (2013) and HL14. HiRAM uses the hydrostatic finite-volume cubed-sphere dynamical core described in Putman and Lin (2007), Harris and Lin (2013), and references therein. This dynamical core solves the hydrostatic primitive equations, in flux form for scalars (including potential temperature) and in vector-invariant form for momentum, on the cubedsphere grid using the Lin and Rood (1997) algorithm in each horizontal layer extended to apply to a system with active thermodynamics. The finite-volume pressure gradient algorithm of Lin (1997) is used. The fluxes are computed using the piecewise-parabolic method (Colella and Woodward 1984) using the positivity-preserving constraint of Lin and Rood (1996) for air mass and tracers. The implicit diffusion from the piecewise-parabolic fluxes and the monotonicity constraint damps grid-scale noise. Further noise control is performed using fourthorder divergence damping. Vertical mass redistribution is performed by the vertically Lagrangian remapping of Lin (2004), modified as in Harris and Lin (2013) to perform the remapping on temperature instead of total energy. A global energy fixer computes lost total energy from the adiabatic dynamics and uniformly returns the lost energy as heat. All simulations use 32 vertical levels as in Z09, and have a constant-pressure top at $1 \mathrm{hPa}$. 
(a) c384

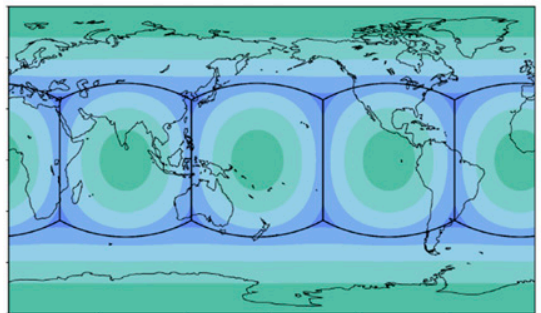

(b) c384r25 OKC

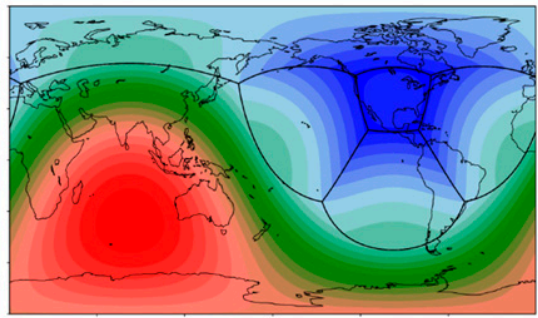

(c) c384r25 Taiwan

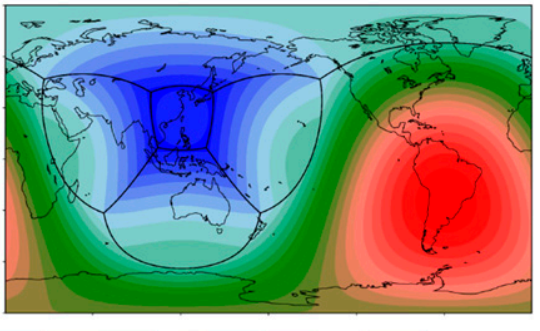

\section{0}

15

20

25

30

35

40

45

50

55

60

65

70

FIG. 1. Grid structure and local gridcell widths ( $\mathrm{km}$; estimated as the square root of the cell area) for comprehensive climate simulations: (a) uniform-resolution c384, (b) OKC stretched c384r25, and (c) Taiwan stretched c384r25. Black lines represent cubed-sphere edges; the smallest outlined region at the center of the "blue flower" in (b) and (c) is the highest-resolution area.

The convective parameterization is the University of Washington shallow convection scheme of Bretherton et al. (2004) extended to represent unresolved deep convection as described in Z09. Microphysics is represented by the six-category bulk microphysical scheme of Lin et al. (1983). The surface flux, boundary layer, orographic gravity wave drag, and radiative transfer parameterizations are the same as in GFDL Atmospheric Model, version 2.1 (AM2.1; Anderson et al. 2004), although the time step at which the radiative tendencies are computed has been decreased from 3 to $1 \mathrm{~h}$, and there is no convective gravity wave drag parameterization. Aerosols, ozone, and well-mixed greenhouse gases are prescribed; there are no interactive aerosols or aerosol indirect effect.

\section{The Schmidt transformation}

The transformation of Schmidt (1977) provides a smooth, analytic means of deforming any spherical grid by "attracting" grid points to a specified central location, which can then be rotated to any other point on the sphere using a solid-body rotation. In this method, grid points of the original cubed sphere are attracted to the South Pole, so that we need only do the stretching along meridians, leaving longitudes unchanged; the South Pole is then moved to the target latitude and longitude by a solid-body rotation. The transformation of the latitude $\theta$ to $\vartheta$ by stretching is given by

$$
\sin \vartheta=\frac{D+\sin \theta}{1+D \sin \theta} \quad \text { with } \quad D=\frac{1-c^{2}}{1+c^{2}}
$$

where $c$ is the stretching factor, which can be any positive real number. The stretching is smooth, so that the gridcell width varies smoothly outward from the refined region, unlike the abrupt refinement resulting from grid nesting. However, the Schmidt transformation also leaves a lower-resolution region on the opposite side of the sphere, which covers more than half of the earth's area. (We will find that this degraded-resolution area does not adversely affect the quality of HiRAM's global climate.) The outline of the resulting stretched grid resembles a "flower" with four petals, the center of which is the highest-resolution area.

The difference between stretched and (quasi-) uniform resolution can be seen in Fig. 1, in which an undeformed c384 $(25 \mathrm{~km})$ grid (Fig. 1a) is deformed by a factor of 2.5 and rotated to two different locations, Oklahoma City $\left(\mathrm{OKC} ; 35.4^{\circ} \mathrm{N}, 97.6^{\circ} \mathrm{W}\right.$; Fig. $\left.1 \mathrm{~b}\right)$ and Taiwan $\left(24.0^{\circ} \mathrm{N}\right.$, $121.0^{\circ} \mathrm{E}$; Fig. 1c). Here, the notation $\mathrm{c} N$ refers to a cubedsphere grid with $N \times N$ grid cells on each of its six faces, and $\mathrm{cNr} T$ refers to a $\mathrm{cN}$ grid with a stretching factor of $T$. Stretching the grid by a factor of 2.5 makes the smallest grid cell about $10 \mathrm{~km}$ wide; alternately, the widest grid cell on the opposite side of the sphere becomes about $75 \mathrm{~km}$ wide. Since Oklahoma City and Taiwan lie on roughly opposite sides of the world, North America lies in the low-resolution region of the Taiwan grid and the highresolution region of the OKC grid, and South and East Asia lie in the high-resolution region of the Taiwan grid and the low-resolution region of the OKC grid. This symmetry will be exploited later to more clearly tie the effects of differing resolution on simulation quality in our comprehensive climate simulations.

The variation of gridcell width with distance from the refined region can be seen in Fig. 2, depicting the equatorial gridcell widths of three uniform-resolution grids (c90, c256, and c768 of average gridcell widths of 103,36 , and $12 \mathrm{~km}$, respectively) and a c256r3 grid with a refined $12-\mathrm{km}$ region centered over the equator. Not only can the variation of the uniform-resolution grid spacing between the face centers and edges be seen, but also the smooth variation of the stretched grid, which varies from a resolution equivalent to that in the c768 grid in its high-resolution region to that comparable to the $\mathrm{c} 90$ grid on the opposing side. The resolution also 


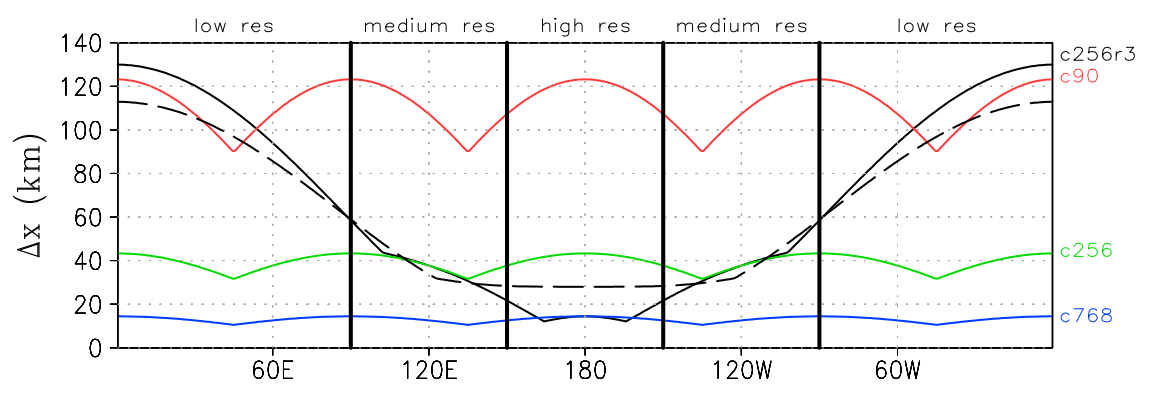

FIG. 2. Plot of the gridcell width along the equator for various cubed-sphere grids used in the aquaplanet simulations: c256r3 (black), c90 (red), c256 (green), and c768 (blue). Labeling at the top denotes regions used for averaging c256r3 results for comparison to the uniform-resolution grids. The dashed black line represents gridcell width in the $\mathrm{c} 256 \mathrm{r} 3$ grid at $40^{\circ}$ latitude. Longitudes are given relative to the location of the high-resolution region.

decreases poleward from the refined region: at $40^{\circ}$ latitude poleward in either direction of the highest-resolution region, the gridcell width is more comparable to that of a c256 grid (dashed line in Fig. 2). Note that the variation in gridcell width at the corners of the stretched grid is slightly smoother than that of the uniform-resolution grid. The relative simplicity of these grids and their refinement over the equator makes them ideal for studying tropical precipitation and tropical cyclones in aquaplanet simulations.

The effect of variable resolution is shown in Fig. 3, which depicts instantaneous outgoing longwave radiation colored to resemble an infrared satellite image. The aquaplanet AP-c768 simulation (Fig. 3a) depicts finescale structure throughout the tropics: convective cloud clusters, tropical cyclones, and two intense tropical cyclones with well-defined eyes. Similar fine structures, including some tropical cyclones and their rainbands, are seen in and near the high-resolution region of the AP-c256r3 simulation (Fig. 3b), indicated by the smallest grid face, but in the coarse grid face (now covering more than half of the domain) cloud clusters are much less well defined. An August snapshot from the comprehensive-climate Taiwan simulation (Fig. 3c) shows a typhoon between Taiwan and the Korean peninsula, while a February image from the OKC simulation (Fig. 3d) shows cloud streets off of the eastern coast of the United States with much finer detail than similar clouds in the Pacific Ocean.

\section{Perpetual-September aquaplanet simulations: Evaluation against uniform-resolution simulations}

Aquaplanet simulations, which will be referred to by their resolution and the prefix AP to distinguish them from the comprehensive climate simulations described in the next section, are HiRAM simulations in which the land model is disabled, the lower boundary is specified by zonally uniform SSTs representative of September observed SSTs (Fig. 4), and there is no topography or orographic gravity wave drag. Here, we are primarily interested in using the aquaplanet as a way to test the quality of the stretched-grid simulation against uniformresolution simulations, and to determine if the representation of precipitation and tropical cyclones is similar in each of the regions of roughly uniform resolution (Fig. 2) to those in uniform-resolution simulations of corresponding resolution, instead of demonstrating a model intended for climate dynamics research. The use of September-like SSTs also differs from the typical aquaplanet simulation design (cf. Blackburn et al. 2013) and is done to ensure a large sample of tropical cyclones to test the model. The performance of HiRAM in standardized comparisons between aquaplanet models can be seen in Blackburn et al. (2013) and Williamson et al. (2013). Characteristics of HiRAM's tropical wave activity and spectrum, of importance for the distribution of tropical precipitation and of the formation of tropical cyclones, are discussed in HL14 and from a coupled version of HiRAM in Xiang et al. (2015).

Four 6-yr-long aquaplanet simulations were performed, after allowing a 1-month spinup period from a dry, resting atmosphere. Three uniform-resolution simulations were performed, one each on the c90, c256, and c768 grids, and a c256r3 simulation with the refined region centered over the equator (Figs. 2 and 3a). These simulations were identical except for their time steps (Table 1) and the fact that AP-c768 uses a 50\% larger value of the autoconversion threshold ( $3 \mathrm{vs} 2 \mathrm{~g} \mathrm{~kg}^{-1}$ ) in the convective scheme, following the recommendation of Z09 to increase the autoconversion threshold with increasing resolution; as in Z09, the convection scheme simply removes all condensate above the autoconversion threshold as precipitation falling immediately to the 

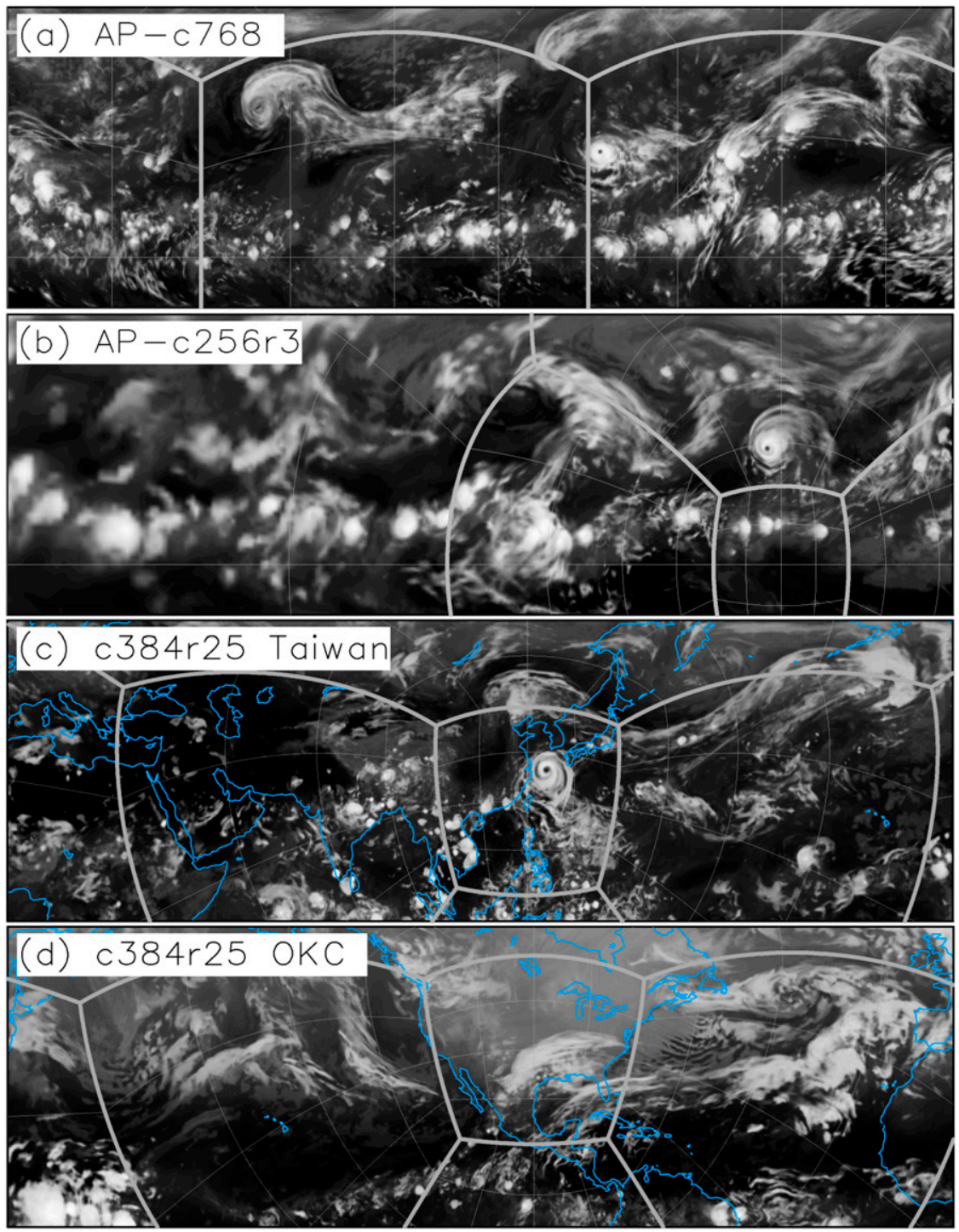

FIG. 3. Examples of instantaneous outgoing longwave radiation; darker shading indicates warmer temperatures as in an infrared satellite image. Heavy white lines delineate cubedsphere tile boundaries. All panels show a $220^{\circ}$ longitude-wide region spanning from the equator to $50^{\circ} \mathrm{N}$. (a) AP-c768 quasi-uniform resolution. (b) AP-c256r3 stretched-grid simulation. (c) The c384r25 Taiwan stretched-grid climate simulation in August. (d) The c384r25 OKC stretched-grid climate simulation in February. The blue-outlines in (c),(d) are land areas.

surface. Insolation is equinoctial with a solar constant of $1365 \mathrm{~W} \mathrm{~m}^{-2}$. Ozone and well-mixed greenhouse gases are fixed at 1980 levels.

\section{a. Precipitation}

The width and intensity of the intertropical convergence zone is known to be a function of model resolution: higher-resolution simulations typically have more intense, narrower ITCZs (cf. Lorant and Royer 2001; Williamson 2008; Rauscher et al. 2013), although this is not universally true (Tomita et al. 2005). The latitude of the ITCZ is also a function of resolution in our perpetualSeptember simulations: the ITCZ is located over the warmest SSTs north of the equator, and at higher resolutions the ITCZ is found closer to the equator (Fig. 5).

The position, intensity, and width of the ITCZ vary smoothly in the variable-resolution AP-c256r3 (Fig. 5a), being the narrowest and closest to the equator in the high-resolution region and then the broadest, weakest, and farthest poleward in the low-resolution region. Artifacts due to the grid edges are not apparent; however, 


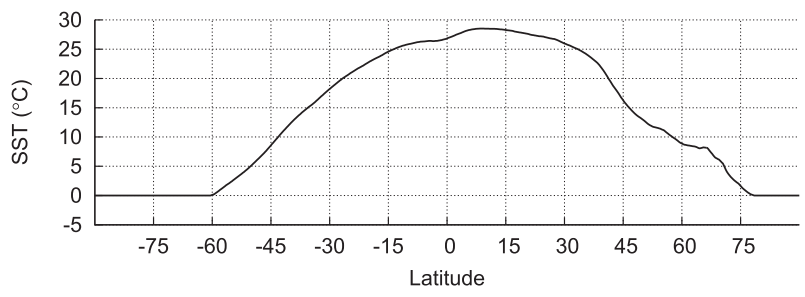

FIG. 4. Zonally uniform September-like SSTs used in aquaplanet simulations.

even the mean precipitation field shows small-scale structure despite the averaging period. Small-scale structure is more visible in the subtropics in Fig. S1 of the supplemental material, showing the same data but with a rescaled color bar. The spatial variability in the precipitation field is due to the substantial small-scale variability in HiRAM: as the convective parameterization is less intrusive than traditional convection schemes (Z09), much of the convection is handled by the resolved-scale dynamics, permitting the formation of many small but organized storms. This activity can be seen in the instantaneous longwave radiation field in Figs. $3 \mathrm{a}$ and $\mathrm{b}$ as well as in 3 -h accumulated precipitation (Fig. S2 of the supplemental material). The variation in the ITCZ location and strength does create artificial zonally asymmetric circulations suggestive of the observed Walker circulation in the equatorial Pacific, as seen by the maximum in precipitation between $60^{\circ}$ and $150^{\circ} \mathrm{E}$ (black shading), which is not repeated to the east of the high-resolution region. Examination of the $0^{\circ}-$ $15^{\circ} \mathrm{N}$ latitude mean 500 -hPa vertical pressure velocity in AP-c256r3 (Fig. 6) finds enhancement of ascent by nearly a third compared to AP-c768 and AP-c256 (about -50 to $-40 \mathrm{hPa} \mathrm{day}^{-1}$ ) between $60^{\circ}$ and $150^{\circ} \mathrm{E}$, and a smaller decrease near $180^{\circ}$ longitude. Similar Walker-type circulations were found in the variable-resolution simulations of Lorant and Royer (2001) and Rauscher et al. (2013).

The behavior of the ITCZ with changing resolution in AP-c256r3 is qualitatively similar to that in the uniformresolution simulations (Figs. 5b,c), in which the highestresolution AP-c768 has the narrowest and farthest equatorward ITCZ; the ITCZ is slightly broader and farther poleward in AP-c256, and weaker, broader, and farther poleward still in AP-c90. The location of the precipitation peak in the high-resolution region matches well that of AP-c768 (Fig. 7a) and the location of the precipitation peak in the low-resolution region matches that of AP-c90 (Fig. 7c), although the precipitation amounts do not precisely match. In the low-resolution region even if a narrower region $\left(50^{\circ} \mathrm{W}-50^{\circ} \mathrm{E}\right.$; dotted line in Fig. 7c) is used to compute the average, in order to more strictly restrict the region to low-resolution regions, there is still more precipitation in the lowresolution region of the stretched grid than in AP-c90, an amount closer to that at higher resolutions. In the medium-resolution region the location and amplitude of the precipitation peak is similar to but farther poleward and lower than AP-c256 (Fig. 7b); taking an average over a wider region $\left(50^{\circ}-150^{\circ} \mathrm{E}\right.$ and $50^{\circ}-150^{\circ} \mathrm{W}$, dotted line), however, yields an excellent match to AP-c256.

The global- and tropical-mean precipitation is roughly the same in all simulations, and in all resolution regions of AP-c256r3 (Table 2); the difference between the largest and smallest values is less than $4 \%$ globally and $7 \%$ in the tropics. The total tropical precipitation is actually less in the high-resolution region of AP-c256r3 than in the lower-resolution regions, while it is greatest in the medium-resolution region. This is at least partially attributable to the artificial Walker circulation decreasing rainfall in the high-resolution region and increasing it in the medium-resolution region. There is more precipitation poleward of $10^{\circ} \mathrm{N}$ at lower resolutions (Fig. 7), reducing the difference in area-averaged precipitation between the resolutions. The convective fraction, the percentage of precipitation caused by the convective scheme (Table 2), shows little dependence upon resolution in AP-c256r3. Only AP-c768 has a significantly lower convective fraction; an AP-c768 simulation with the autoconversion threshold reduced to the same value as in the other simulations (from 3 to $2 \mathrm{~g} \mathrm{~kg}^{-1}$ ) only increased the convective fraction to $9 \%$ from 7\%, but an AP-c256r3 simulation with a threshold of 3 instead of $2 \mathrm{~g} \mathrm{~kg}^{-1}$ had a convective fraction of less than $10 \%$ in all regions. On the AP-c90 grid, the convective fraction varied from $9 \%$ when using a threshold of $3 \mathrm{~g} \mathrm{~kg}^{-1}$ to over $80 \%$ when using a threshold of $1 \mathrm{~g} \mathrm{~kg}^{-1}$. We conclude that convective fraction is relatively insensitive to resolution and more sensitive to tuning parameters such as the autoconversion threshold,

TABLE 1. Simulation parameters: average grid spacing (for uniform-resolution simulations) and the physics and dynamics time steps.

\begin{tabular}{lcccccc}
\hline \hline & AP-c768 & AP-c256 & AP-c90 & AP-c256r3 & c384 & c384r25 \\
\hline Average $\Delta x(\mathrm{~km})$ & 12 & 36 & 103 & $10-130$ & 25 & $10-75$ \\
Physics $\Delta t(\mathrm{~s})$ & 300 & 450 & 1800 & 450 & 300 & 300 \\
Dynamics $\Delta t(\mathrm{~s})$ & 15 & 45 & 150 & 22.5 & 30 & 12.5 \\
\hline
\end{tabular}



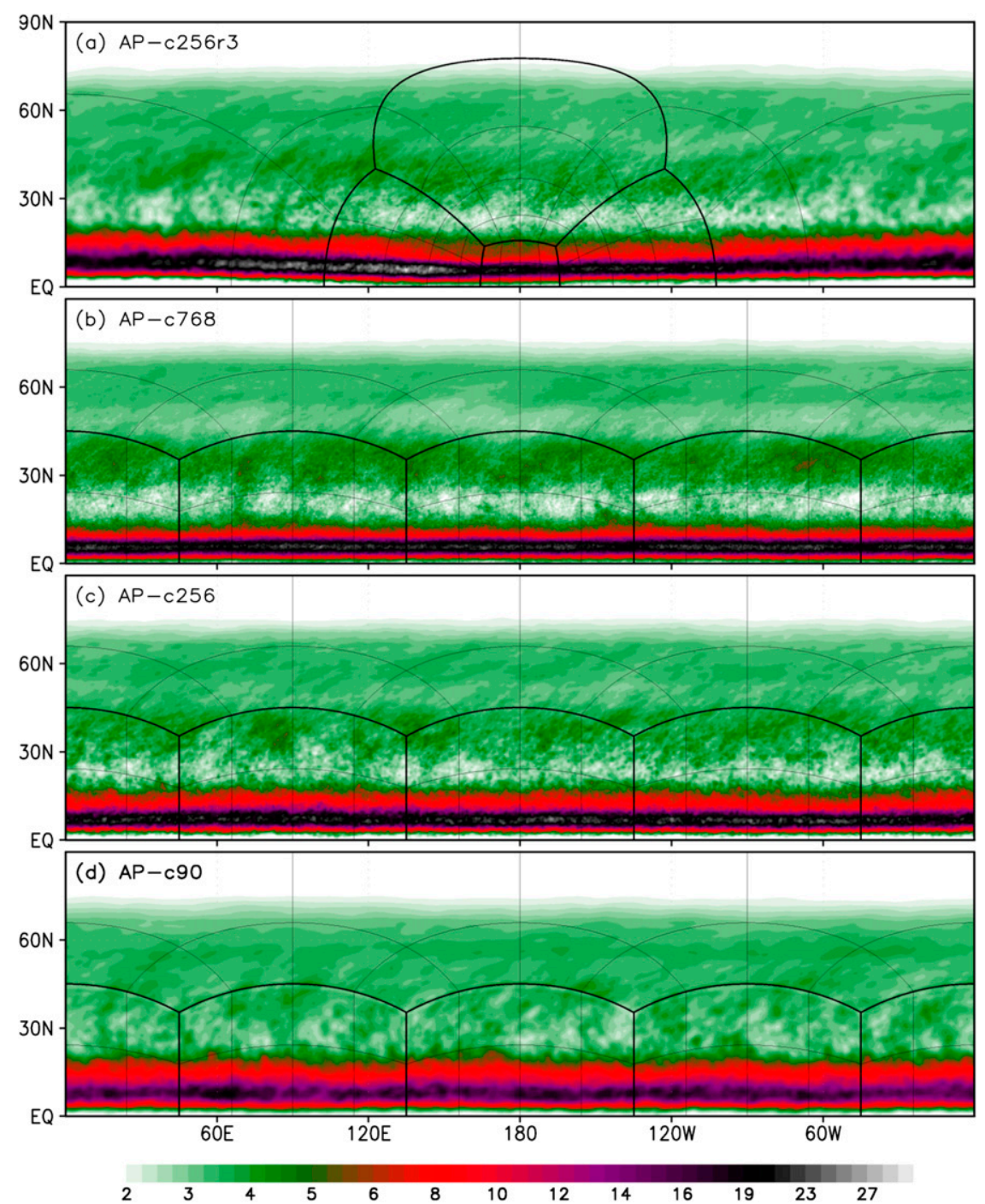

FIG. 5. Average precipitation $\left(\mathrm{mm} \mathrm{day}^{-1}\right)$ in 3-yr aquaplanet simulations with fixed September SST: (a) AP-c256r3, (b) AP-c768, (c) AP-c256, and (d) AP-c90. Black lines represent cubedsphere edges.

until convection-permitting scales near $12 \mathrm{~km}$ are achieved, at which the convective parameterization is less important and more convection is explicitly resolved.

\section{b. Tropical cyclones}

Resolution has interesting and sometimes counterintuitive effects upon the numbers of tropical cyclones and the fraction attaining hurricane strength. Long-lived tropical cyclones (LLTCs) are counted and classified in the 6-hourly output from our simulations by a simple cyclone tracker. This tracker identifies warm-core cyclones represented by sea level pressure (SLP) minima as TCs if the longevity requirements of Chen and Lin (2011, 2013) are satisfied: the total lifetime of an identified cyclone (regardless of wind speed or warm core) must be at least $72 \mathrm{~h}$, the cyclone must have a warm core for a cumulative (not necessarily consecutive) period of $48 \mathrm{~h}$, and the maximum $10-\mathrm{m}$ wind speed must be greater than $17.5 \mathrm{~m} \mathrm{~s}^{-1}$ and must coexist with a warm core for 36 consecutive hours. The longevity requirement removes many short-lived and disorganized storms that are difficult to track. A warm-core system is classified as a hurricane-strength storm if the $10-\mathrm{m}$ wind speed reaches $32.5 \mathrm{~m} \mathrm{~s}^{-1}$ at a location equatorward of $40^{\circ}$ latitude at least once during its lifetime. For brevity we will refer to any hurricane-strength storm simply as a hurricane, except when specifically discussing typhoons in the western Pacific and severe cyclones in the Indian 


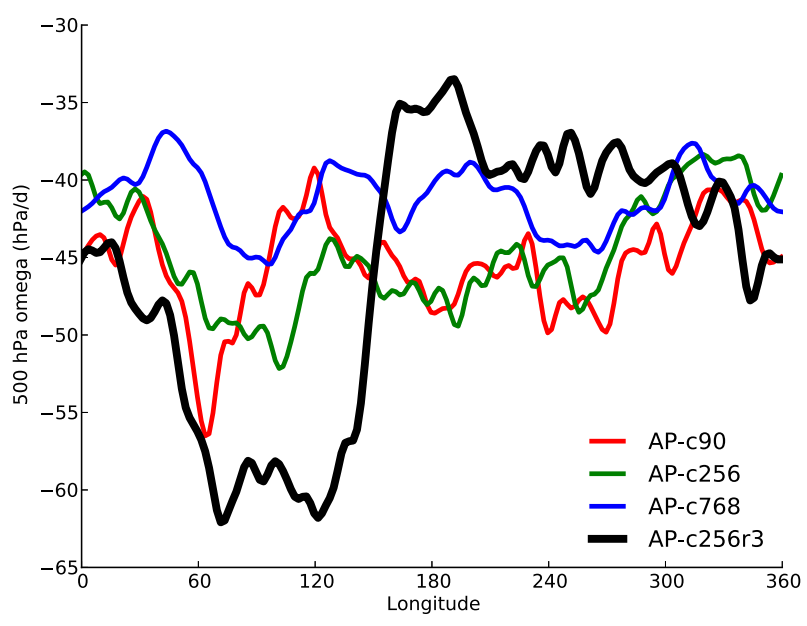

FIG. 6. The $0^{\circ}-15^{\circ} \mathrm{N}$ mean $500-\mathrm{hPa}$ vertical pressure velocity $\left(\mathrm{hPa} \mathrm{day}^{-1}\right)$ in aquaplanet simulations.

Ocean and Southern Hemisphere. More details of the tracking algorithm are given in the appendix. We do not adjust the wind thresholds to compensate for lower resolutions, nor do we use an empirically adjusted lowest-level or 850 -hPa wind as a proxy for $10-\mathrm{m}$ winds, but instead use explicitly computed 10-m winds from the boundary layer scheme. Discussions on the realism of tropical cyclones in global models are given by Walsh et al. (2007) and Halperin et al. (2013), and references therein.

The TC storm density, given in terms of cumulative days that a cyclone is found in a given $5^{\circ} \times 4^{\circ}$ box (Fig. 8), shows a smooth variation in AP-c256r3 from relatively high values in the low-resolution region to smaller numbers in the high-resolution region. There are fewer storms in the high-resolution region than in the low-resolution region (Table 3). There are about 50\% more TCs in AP-c256 than in AP-c768, which in turn has half as many TCs as does AP-c90. Longitudinally averaged track densities (Fig. 9) show good agreement between TCs in the uniform-resolution simulations (solid colored lines) and the corresponding region of the stretched-grid simulation (solid black lines).

The storm density (Fig. 8) of hurricane-strength storms is greatest in the medium-resolution region of AP-c256r3, and in AP-c256. However, the fraction of TCs attaining hurricane strength (Table 3) is greatest in the highresolution region of AP-c256r3, and in AP-c768; this fraction increases monotonically with resolution, although it does so more strongly in the uniform-resolution simulations than in the stretched-grid simulation.

Two competing factors determine the number of hurricanes in our simulations: the number of TCs, and the ability of the model to resolve intense cyclones. Both Merlis et al. (2013) and Ballinger et al. (2015) found a relation between TC frequency and the ITCZ latitude in their aquaplanet HiRAM simulations; we follow their argument here. TCs are known to develop most frequently at the poleward side of the ITCZ (Gray 1968); since at higher resolution the ITCZ is located closer to the equator, then the region for best cyclogenesis occurs nearer to the equator, where the Coriolis force needed to organize convection into cyclones is weaker. The $f$-plane experiments of Held et al. (2007) found that there are fewer TCs when the Coriolis parameter is reduced, with the number of TCs halved when the ambient rotation was moved from $10^{\circ}$ to $5^{\circ}$ latitude; similar results were found by Zhou et al. (2014). Thus we expect there to be fewer TCs at the higher resolutions due to the equatorward shift of the ITCZ. The tropical maximum in TC storm density shifts equatorward along with the ITCZ at increasing resolution (Fig. 9).

Alternately, the features in TCs representing the strongest wind speeds are better resolved as the model resolution increases. Lower-resolution models do not produce as many intense TCs as do higher-resolution models (e.g., Z09; Walsh et al. 2007; Bender et al. 2010; Zhao and Held 2010, and references therein), making the wind speed requirements for both TCs and hurricanes harder to meet.

How can the effects of intensity and storm frequency on resolution be separated? The wind speed criterion can be replaced with an SLP criterion, which is known to be less sensitive to resolution in HiRAM (cf. Chen and Lin 2013). The low-resolution regions of AP-c256r3 and AP-c90 still produce many storms of deep central pressures (Fig. 10), but very few of these storms have maximum winds exceeding $40 \mathrm{~m} \mathrm{~s}^{-1}$. In contrast, AP-c768 and the high-resolution region of AP-c256r3 show a nearly linear pressure-wind relation to storms as intense as $50 \mathrm{~m} \mathrm{~s}^{-1}$ and as deep as $920 \mathrm{hPa}$. Replacing the wind criterion with a pressure criterion-that a TC must have a central SLP no greater than $1000 \mathrm{hPa}$-in the longevity requirements makes the increase in the number of TCs with resolution even stronger than with the original wind criterion (Table 3), more clearly showing the greater number of TCs produced at lower resolutions. If the threshold for hurricane-strength storms is changed to having a minimum SLP less than $980 \mathrm{hPa}$, the increase with resolution in the fraction of storms attaining hurricane-strength is much less than if the wind speed criterion is used.

The high-resolution region of AP-c256r3 has fewer overall TCs than does AP-c768 (Fig. 9), both in the tropics and subtropics, and the minimum in storm density is shifted westward from the center of the highresolution region (Fig. 8). This off-center storm density minimum corresponds to the descent in the artificial 


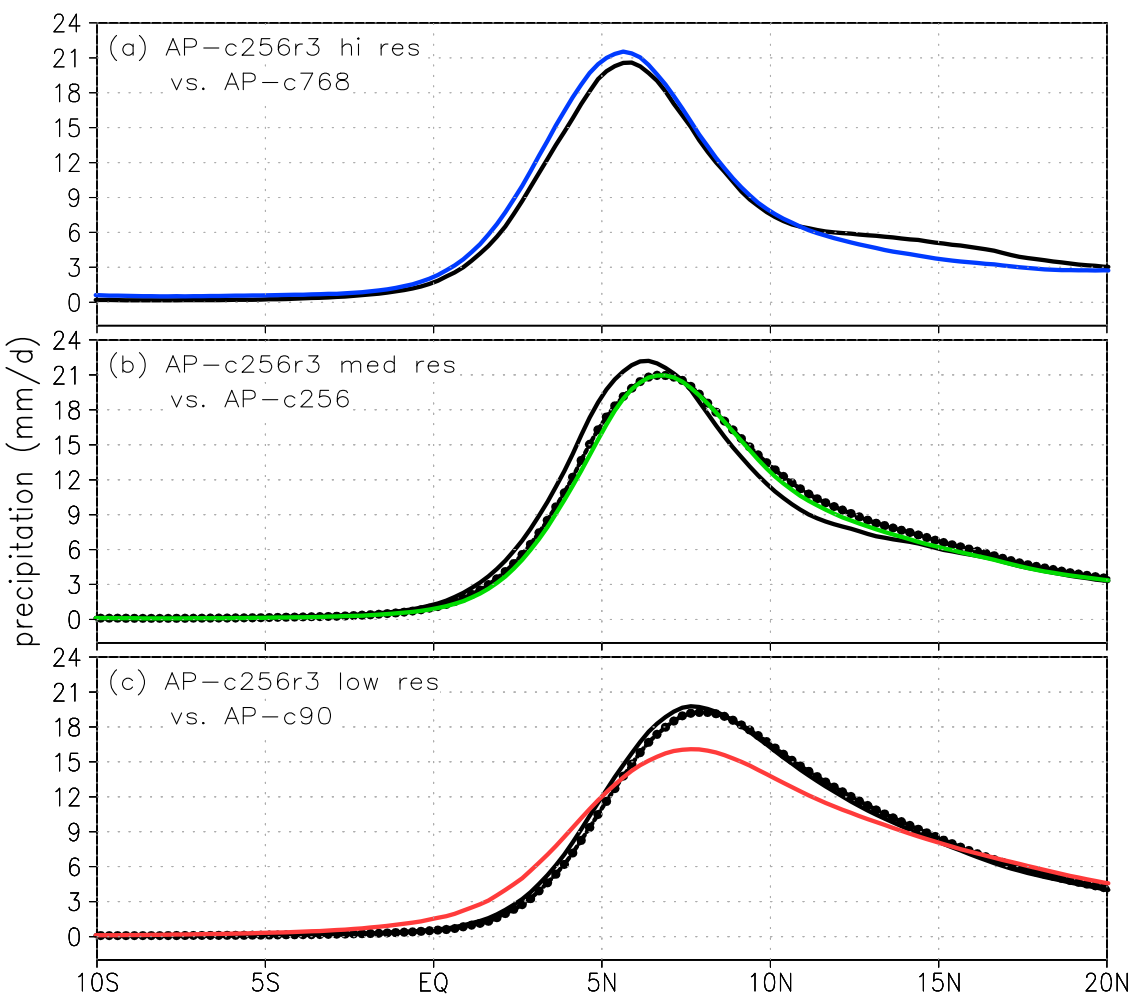

FIG. 7. Longitudinally averaged precipitation $\left(\mathrm{mm} \mathrm{day}^{-1}\right)$ in 3-yr aquaplanet simulations with fixed September SST. Zonal means are shown for the uniform-resolution simulations; regional means (extents shown in Fig. 2) are shown for the AP-c256r3 simulation. (a) AP-c768 (blue) vs high-resolution AP-c256r3 (black) region. (b) AP-c256 (green) vs medium-resolution AP-c256r3 (black) region. (c) AP-c90 (red) vs low-resolution AP-c256r3 (black) region. In (b) and (c), the black dotted lines are the AP-c256r3 simulation in which the low-resolution region is reduced to $50^{\circ} \mathrm{W}-50^{\circ} \mathrm{E}$ and the medium-resolution expanded to $50^{\circ} \mathrm{E}-150^{\circ} \mathrm{E}$ and $50^{\circ} \mathrm{W}-150^{\circ} \mathrm{W}$.

Walker circulation (Fig. 6) and may be suppressing cyclone activity in this region.

\section{AMIP simulation using HiRAM and comparison with observations}

Three AMIP comprehensive climate simulations for 1981-2008 were performed, one for each of the c384, c384r25 OKC, and c384r25 Taiwan grids. The c384 simulation is very similar to the c360 simulations, which have been used for a number of studies (Chen and Lin 2011, 2013; Gall et al. 2011), and was used for both present-day and future time-slice integrations submitted to the CMIP5 archive (Z09).

The model configuration was largely the same in all three simulations, with minor differences to suit the locally enhanced resolution. The physics time step in the stretched simulations was decreased from 600 to $300 \mathrm{~s}$; the dynamics time step was decreased from 30 to $12.5 \mathrm{~s}$; and the nondimensional scaling parameter for the cumulus horizontal entrainment rate $\left[e_{0}\right.$ in Zhao et al. (2012)] was increased from 10 to 12 , which acts to increase the intensity of convectively coupled waves and tropical cyclones. Otherwise, the parameter tuning for

TABLE 2. Aquaplanet simulations: Average precipitation and convective fraction. Labels with high, med, and low refer to the regions in Fig. 2.

\begin{tabular}{|c|c|c|c|c|c|c|c|}
\hline Precip $\left(\mathrm{mm}\right.$ day $\left.^{-1}\right)$ & AP-c768 & $\begin{array}{c}\text { AP-c256r3 } \\
\text { high }\end{array}$ & AP-c256 & $\begin{array}{c}\text { AP-c256r3 } \\
\text { med }\end{array}$ & AP-c90 & $\begin{array}{c}\text { AP-c256r3 } \\
\text { low }\end{array}$ & $\begin{array}{c}\text { AP-c256r3 } \\
\text { global }\end{array}$ \\
\hline $90^{\circ} \mathrm{N}-90^{\circ} \mathrm{S}$ & 3.50 & 3.45 & 3.53 & 3.60 & 3.48 & 3.48 & 3.51 \\
\hline $30^{\circ} \mathrm{N}-30^{\circ} \mathrm{S}$ & 4.04 & 3.86 & 4.08 & 4.18 & 4.04 & 4.11 & 4.09 \\
\hline Convective fraction & 7.2 & 27.6 & 25.7 & 25.5 & 28.3 & 22.4 & 24.3 \\
\hline
\end{tabular}



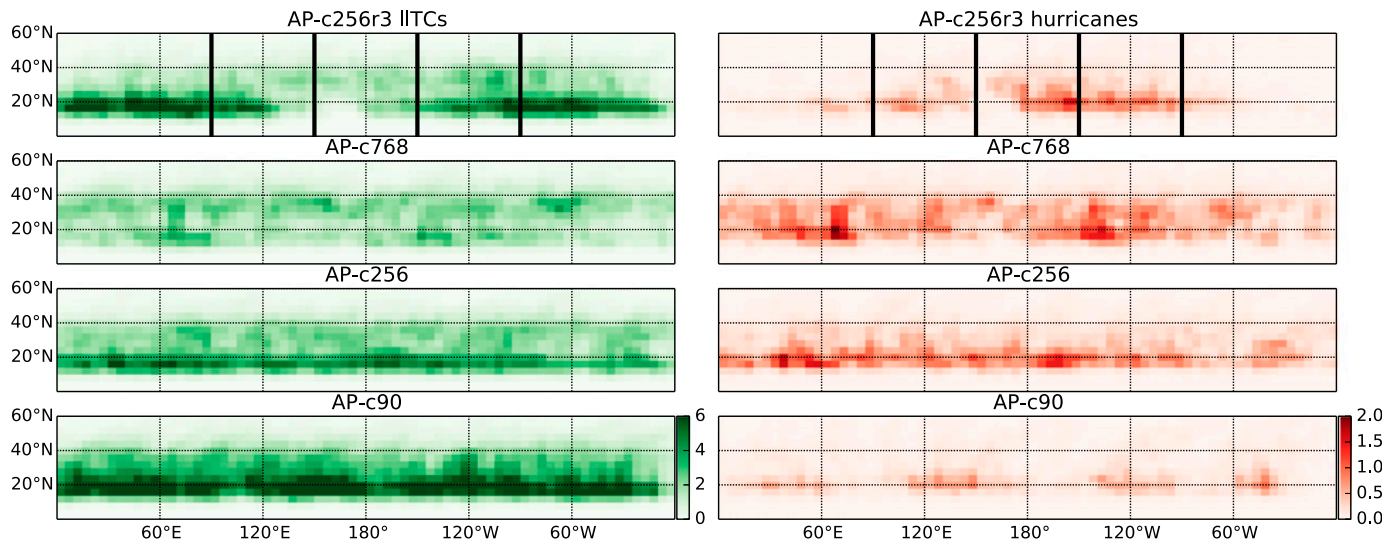

FIG. 8. Track densities (storm days per 12 months) of (left) LLTCs and (right) hurricanes in (top)-(bottom) aquaplanet simulations in $5^{\circ}$ lon $\times 4^{\circ}$ lat bins: AP-c256r3, AP-c768, AP-c256, and AP-c90. the stretched-grid simulations was the same as for c384, as the tuning for the $\mathrm{c} 384$ could be considered a "compromise" between the low- and high-resolution regions of the stretched grid simulations. Tuning in the two stretched simulations was identical, except that in the orographic gravity wave drag parameterization (Stern and Pierrehumbert 1988) the empirical scaling parameter [ $G^{*}$ in Eq. (4) of Anderson et al. (2004)] for the maximum permitted unresolved wave drag was set to 4 in the OKC simulation and 3 in the Taiwan and c384 simulations. The OKC simulation has better resolution of the Rockies, a major orographic wave source, and so a larger value of the scaling parameter is needed.

The simulations described in this section use sea surface temperatures and sea ice specified from the Hadley Centre Sea Ice and Sea Surface Temperature (HadISST; Rayner et al. 2003) 1-degree monthly dataset. The GFDL Land Model, version 3 (LM3; Milly et al. 2014), with specified daily vegetation is used on the land surface, although due to technical and personnel limitations the river module has been disabled. These simulations used the same time-varying greenhouse gas and aerosol concentrations and solar forcing as described by Z09, but without volcanic aerosols. A $2 \Delta z$ Richardson numberbased local mixing is applied as a "safety valve" to filter $2 \Delta z$ shear instabilities and alleviate gridpoint storms. This method is less intrusive than traditional nonlocal mixing schemes but is not intended to replace the existing boundary layer scheme.

\section{a. Global climate}

Global-mean measures of the radiation budget, water vapor, and precipitation are given in Table 4. None of the quantities varies greatly between the simulations and all are largely comparable to those of the simulations in HL14 (see their Table 2), although the global precipitation amount and both absorbed shortwave and outgoing longwave radiation are all slightly larger and water vapor path slightly lower. All of these values are comparable to those in observations, as discussed in HL14; indeed, the Taiwan stretched-grid simulation, which covers the western Pacific warm pool and the Maritime Continent with its high-resolution region, has a value of the water vapor path very similar to the

TABLE 3. LLTC $\left(>17.5 \mathrm{~m} \mathrm{~s}^{-1}\right)$ and hurricane-strength $\left(>32.5 \mathrm{~m} \mathrm{~s}^{-1}\right)$ storm counts per 12 months of each aquaplanet simulation, the fraction (\%) of all TCs which are hurricane-strength storms, the storm count for LLTCs using a minimum SLP criterion (minimum pressure $<1000 \mathrm{hPa}$ ), and the fraction (\%) of all LLTCs (as counted by the minimum SLP criterion) that have central pressures less than $980 \mathrm{hPa}$. Counts in the AP-c256r3 stretched simulation are weighted by the size of the averaging area (number in parentheses) so as to be comparable to the global uniform-resolution results, and storms are counted in each region based on when a cyclone is first identified. Storm counts are rounded to the nearest integer.

\begin{tabular}{|c|c|c|c|c|c|c|c|}
\hline & AP-c768 & $\begin{array}{c}\text { AP-c256r3 } \\
\text { high (6) }\end{array}$ & AP-c256 & $\begin{array}{c}\text { AP-c256r3 } \\
\text { med (3) }\end{array}$ & AP-c90 & $\begin{array}{c}\text { AP-c256r3 } \\
\text { low (2) }\end{array}$ & $\begin{array}{c}\text { AP-c256r3 } \\
\text { global }\end{array}$ \\
\hline LLTCs & 154 & 122 & 222 & 203 & 298 & 238 & 209 \\
\hline Hurricanes & 85 & 48 & 99 & 73 & 69 & 58 & 62 \\
\hline Hurricane fraction & 55.0 & 39.3 & 44.6 & 35.6 & 23.3 & 24.4 & 29.4 \\
\hline Storm count $<1000 \mathrm{hPa}$ & 177 & 137 & 255 & 240. & 416 & 300. & 253 \\
\hline LLTCs $<980 \mathrm{hPa}$ fraction & 66.8 & 66.0 & 65.2 & 58.5 & 56.9 & 56.1 & 57.7 \\
\hline
\end{tabular}



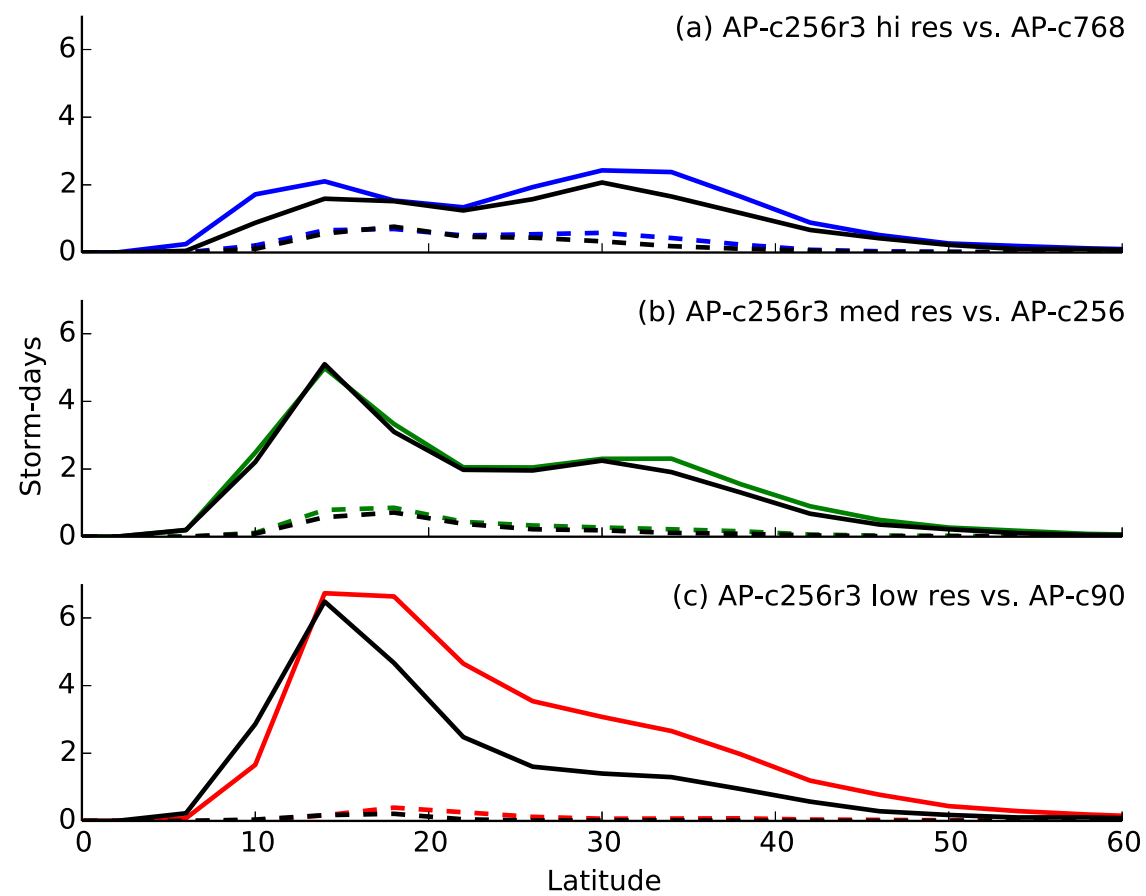

FIG. 9. Track densities (storm days per 12 months) of LLTCs (solid lines) and hurricanestrength storms (dashed lines). Zonal means are shown for the uniform-resolution simulations (colored lines); regional means are shown for the AP-c256r3 simulation (black, extents shown in Fig. 2). (a) AP-c768 vs high-resolution AP-c256r3 region; (b) AP-c256 vs medium-resolution AP-c256r3 region; and (c) AP-c90 vs low-resolution c256r3 region.

$24.7 \mathrm{~kg} \mathrm{~m}^{-2}$ value from the NASA Water Vapor Project (NVAP; Simpson et al. 2001). The tropical-mean precipitation is slightly greater in the OKC simulation than in the other two simulations; all three are larger than from Global Precipitation Climatology Project
(GPCP; Adler et al. 2003) or Tropical Rainfall Measuring Mission version 7 (TRMM v7; Huffman et al. 2007; Wang et al. 2014), which have annual $30^{\circ} \mathrm{N}-30^{\circ} \mathrm{S}$ mean precipitation of 3.39 and $3.27 \mathrm{~mm} \mathrm{day}^{-1}$, respectively.

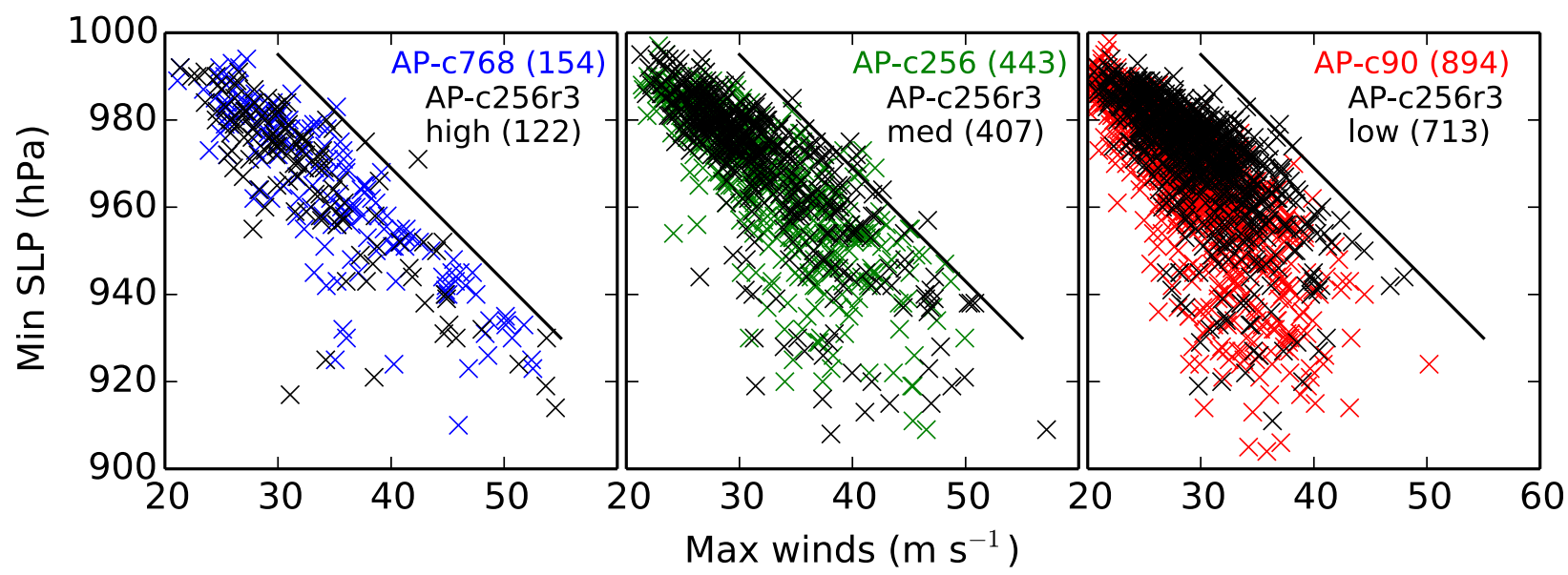

FIG. 10. Maximum wind speed vs minimum sea level pressure for tropical cyclones in aquaplanet simulations. Numbers in parentheses are the 6-yr counts of TCs. Black lines in each panel have slopes of $-3 \mathrm{hPa}\left(\mathrm{m} \mathrm{s}^{-1}\right)^{-1}$. Here, we have sampled a fraction of the storms from the uniform-resolution simulations (every sixth storm from AP-c768; every third storm from AP-c256; every other storm from AP-c90) so that the number of storms is comparable to those in the regions of the stretched-grid simulation: (left) AP-c768 vs high-resolution AP-c256r3 region, (center) AP-c256 vs medium-resolution AP-c256r3 region, and (right) AP-c90 vs low-resolution AP-c256r3 region. 
TABLE 4. Global- and tropical-mean $\left(30^{\circ} \mathrm{N}-30^{\circ} \mathrm{S}\right)$ precipitation, annually averaged values of water vapor path (WVP), net absorbed shortwave radiation (SW abs. rad.), outgoing longwave radiation (OLR), and net top-of-the-atmosphere radiation (net radiation) in HiRAM climate simulations.

\begin{tabular}{lccc}
\hline \hline & & \multicolumn{2}{c}{ c384r25 } \\
\cline { 3 - 4 } & c384 & OKC & Taiwan \\
\hline Global precipitation $\left(\mathrm{mm} \mathrm{day}^{-1}\right)$ & 2.91 & 2.93 & 2.91 \\
Tropical precipitation $\left(\mathrm{mm} \mathrm{day}^{-1}\right)$ & 3.52 & 3.59 & 3.53 \\
WVP $\left(\mathrm{kg} \mathrm{m}^{-2}\right)$ & 25.0 & 24.9 & 24.6 \\
SW abs. rad. $\left(\mathrm{W} \mathrm{m}^{-2}\right)$ & 237.9 & 237.6 & 238.1 \\
OLR $\left(\mathrm{W} \mathrm{m}^{-2}\right)$ & 236.0 & 236.6 & 236.6 \\
Net radiation $\left(\mathrm{W} \mathrm{m}^{-2}\right)$ & 1.84 & 0.98 & 1.48 \\
\hline
\end{tabular}

Despite the coarser resolution covering much of the earth (Fig. 1) we find that the stretched-grid simulations have smaller globally-averaged errors for dynamical fields such as zonal-mean annual winds (Fig. 11) and annual Northern Hemisphere SLP (Fig. 12). In particular, the OKC simulation has root-mean-square errors (RMSEs; Table 5) of just over $1 \mathrm{~m} \mathrm{~s}^{-1}$ for zonal-mean wind and $1 \mathrm{hPa}$ for Northern Hemisphere SLP, owing largely to a decrease in stratospheric wind biases in the tropics and Southern Hemisphere, and to substantial decreases in Arctic and North Atlantic pressure biases. All three simulations have very good RMSEs for winds and (except for c384) SLP compared to the $2^{\circ} \mathrm{AM} 2$ and AM 3 as well as the $1 / 2^{\circ}$ HiRAM simulations of Z09 (c180) and HL14 (c192).

Conversely, the zonal-mean temperature errors increase in the stretched-grid simulations (Table 5) owing mostly to increased errors in the stratosphere and especially in the Antarctic (Fig. 13) where both stretchedgrid simulations have their coarsened regions. All simulations have very low temperature biases in the tropics. Degradation of precipitation biases is seen over the Indian Ocean in the OKC simulation, and over South and Central America and the surrounding oceans in the Taiwan simulation (Fig. 14); both correspond to the coarsest regions of their respective grids. The western Pacific wet bias in the uniform-resolution simulation is alleviated in both of the stretched-grid simulations, although whether stretching gets the model closer to the observations here is dependent on the observation dataset, as GPCP and TRMM yield different precipitation amounts in this region.

\section{b. Regional precipitation: Spatial fidelity and diurnal cycle}

Here, we examine precipitation biases with respect to the latest version (v7) of the TRMM (Gopalan et al. 2010; Wang et al. 2014), which has much improved tropical precipitation compared to earlier versions of TRMM. The annual precipitation bias with respect to TRMM in the c384 uniform-resolution simulation (Fig. 15b) has a dipole pattern in the western Pacificwet in the north and dry in the south-which is reversed in the South China Sea and the eastern Indian Ocean, and is enhanced during the summer (JJA) monsoon season (Fig. 15e). A wet bias is apparent in Indochina as well, partially tied to the model placing the precipitation maximum observed over the eastern Bay of Bengal over land. The Taiwan simulation (Figs. 15c,f) replaces the oceanic dipole bias with a more uniform dry bias. The annual wet bias southeast of Japan is substantially alleviated in the Taiwan simulation, as is the wet bias in Indochina, although in the monsoon season there now (a) NCEP Reanalysis

(b) c384

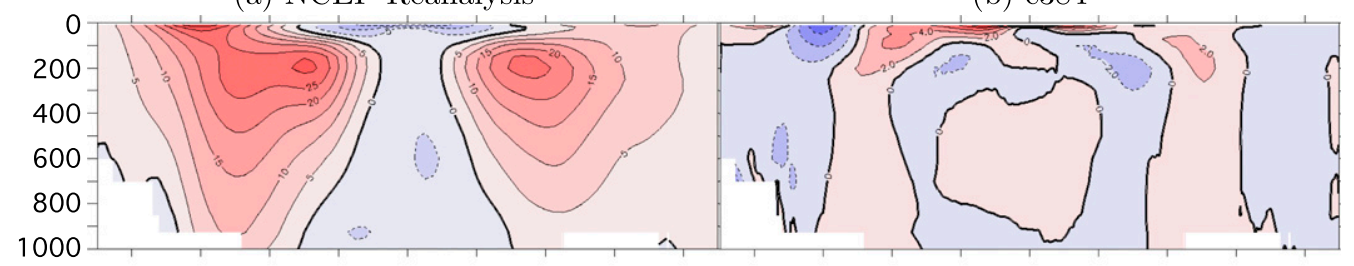

(c) c384r 25 OKC

(d) c384r25 Taiwan

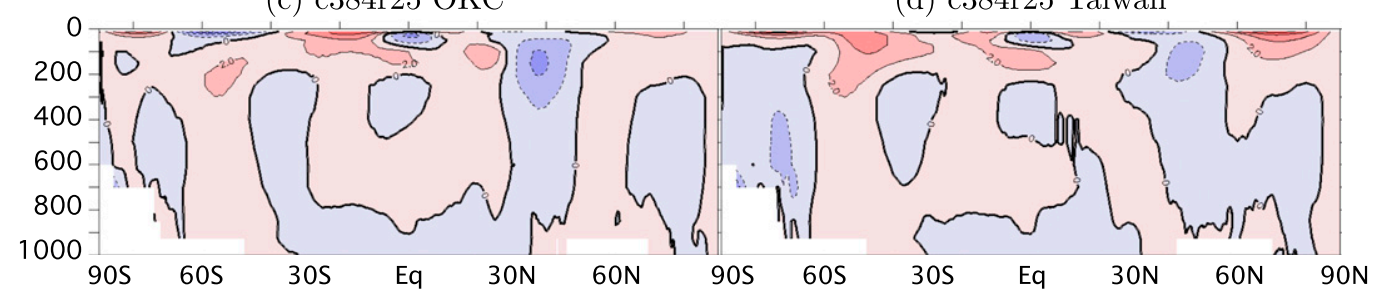

FIG. 11. Zonal-mean winds in HiRAM climate simulations. (a) NCEP reanalysis annual mean winds, contour interval is $10 \mathrm{~m} \mathrm{~s}^{-1}$. Annual mean biases for (b) c384, (c) c384r25 Taiwan, and (d) c384r25 Oklahoma City, contour interval is $2 \mathrm{~m} \mathrm{~s}^{-1}$. In (a)-(d), positive (negative) values are red (blue) and the zero contour is thickened. 
(a) NCEP Reanalysis

(b) c384

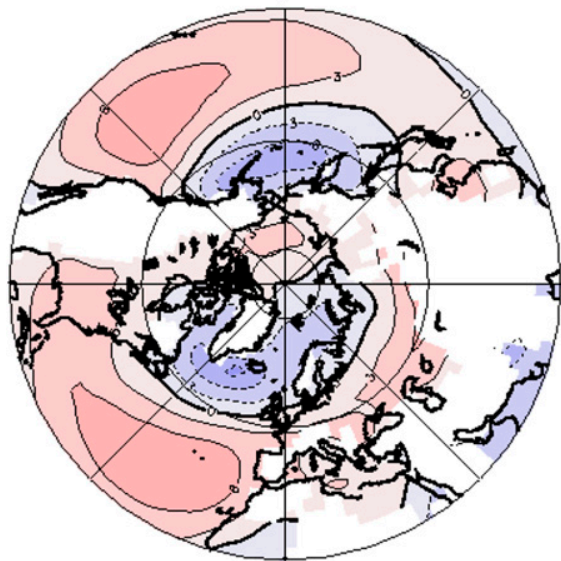

(c) $\mathrm{c} 384 \mathrm{r} 25 \mathrm{OKC}$

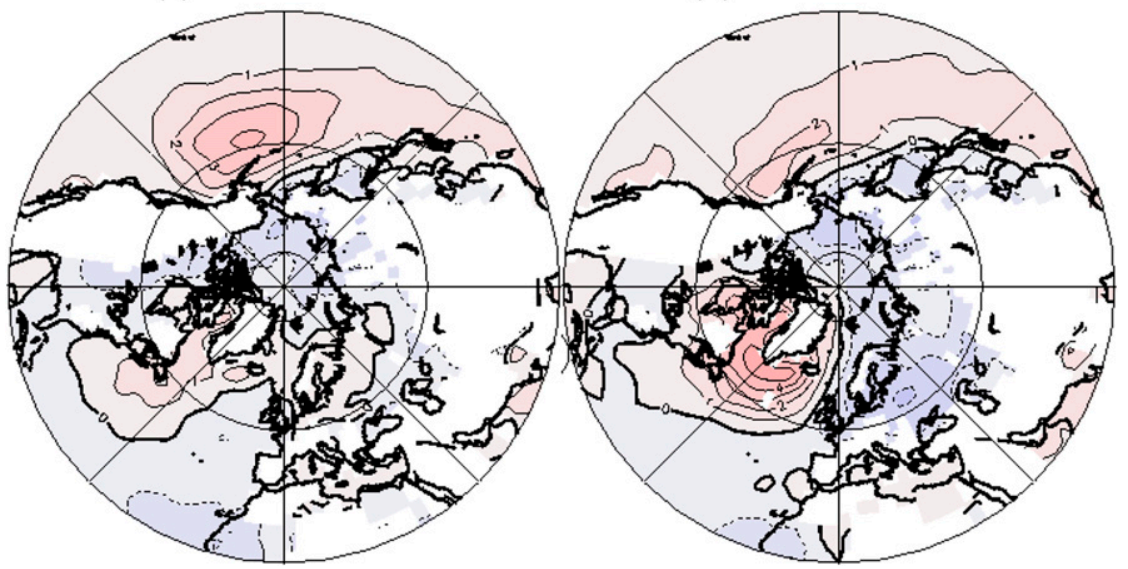

FIG. 12. (a) Annually averaged NCEP reanalysis Northern Hemisphere sea level pressure (3-hPa contours, difference from $1013.25 \mathrm{hPa}$ ). Annual-mean biases (1-hPa contours) for (b) c384, (c) c384r25 Taiwan, and (d) c384r25 Oklahoma City. In (a)-(d), positive (negative) values are red (blue) and the zero contour is thickened.

is a dry bias east of the Philippines. There is also a reduction in the precipitation bias near the Himalayas, in part due to the enhanced resolution of the topography.

The c384 simulation (Fig. 16b) suffers dry biases in the U.S. central plains, the Gulf of Mexico, and the western Atlantic storm track, and wet biases in the eastern United States, the Caribbean, Mexico, Central America, and along the ITCZ in the eastern Pacific, most being more pronounced in the JJA warm season (Fig. 16e). The OKC simulation (Figs. 16c,f) alleviates the biases in the central plains, Atlantic, and Caribbean by a small degree. Improved resolution greatly enhances the detail in orographic precipitation in the mountainous western United States, especially during the DJF cold season when mountains receive the bulk of their precipitation (Fig. 17). Biases in high-resolution orographic precipitation fields over the continental United States can be evaluated against the 4-km Parameter-Elevation
Regressions on Independent Slopes Model (PRISM; Daly et al. 2008) dataset. Many finescale topographic details are visible in the OKC simulation that are not visible in the uniform-resolution simulation, and some dry biases (particularly in Southern California and the Oregon coastal range) are alleviated, although there is now more precipitation than observed in the Sierra Nevada range.

The diurnal cycle of warm-season continental convection (Fig. 18) has been a challenge to climate models, as traditional convective parameterizations cause convection to trigger too early, causing a maximum in precipitation around local noon and early afternoon and a minimum in the early evening, the opposite of what is typically observed. Over the continental United States, the c384 simulation and the Taiwan simulation yield similar JJA diurnal cycles, with the aforementioned erroneous noontime maximum and evening minimum, 
TABLE 5. Global root-mean-square errors, compared to GPCP for precipitation, and NCEP reanalysis for wind, temperature, and SLP, for comprehensive-climate simulations described in this paper, compared to other GFDL simulations.

\begin{tabular}{|c|c|c|c|c|c|c|c|}
\hline & \multirow[b]{2}{*}{ c384 } & \multicolumn{2}{|c|}{$\mathrm{c} 384 \mathrm{r} 25$} & \multirow{2}{*}{$\begin{array}{c}\text { AM2.1 } \\
2^{\circ}\end{array}$} & \multirow{2}{*}{$\begin{array}{l}\text { AM3 } \\
\text { c48 }\end{array}$} & \multirow{2}{*}{$\begin{array}{c}\text { HiRAM } \\
\text { c180 }\end{array}$} & \multirow{2}{*}{$\begin{array}{l}\text { HL14 } \\
\text { c192 }\end{array}$} \\
\hline & & $\mathrm{OKC}$ & Taiwan & & & & \\
\hline Zonal-mean $u\left(\mathrm{~m} \mathrm{~s}^{-1}\right)$ & 1.33 & 1.11 & 1.23 & 1.50 & 1.90 & 1.26 & 1.85 \\
\hline NH SLP (hPa) & 2.04 & 1.06 & 1.39 & 1.83 & 1.47 & 0.85 & 1.42 \\
\hline Zonal-mean $T(\mathrm{~K})$ & 1.29 & 1.63 & 2.14 & 2.06 & 1.83 & 2.00 & 1.67 \\
\hline Precipitation $\left(\mathrm{mm} \mathrm{day}^{-1}\right)$ & 1.04 & 1.30 & 1.24 & 0.85 & 1.06 & 1.07 & 1.01 \\
\hline
\end{tabular}

and an additional maximum at midnight; the c384 simulation yields only a minor "flattening" of this erroneous cycle despite it having better resolution than the Taiwan simulation in this region. However, in the OKC simulation the diurnal cycle is greatly improved: the maximum is now in the late evening, $3 \mathrm{~h}$ after the observed peak, and has a minimum in the early morning; the amplitude of the diurnal cycle is also much closer to that of the observations. A similar improvement is seen over Central America. The Taiwan stretched simulation also yields a greatly improved diurnal cycle averaged over East or Southeast Asia compared to the c384 simulation, which only slightly improved upon the diurnal cycle in the OKC simulation.

While the global- and tropical-mean diurnal cycles for both of the stretched-grid simulations still exhibit an erroneous minimum in the evening, indicating that the improvement is local to the refined region, they do not have the noontime local maximum of the uniform-resolution simulation, possibly indicating that improvement in the higher-resolution regions of the stretched-grid simulations is not cancelled by the lower-resolution regions. The global and tropical diurnal cycles may have been improved in the stretched-grid simulations by the larger entrainment used in their convective parameterizations, so that less precipitation occurs due to the parameterization and thereby can better follow the diurnal cycle. Globally, 21\% of JJA precipitation is from the convective parameterization, compared to only $13 \%$ in each stretchedgrid simulation; in the tropics these fractions increase to $33 \%$ and $21 \%$, respectively, and similar values are found in the regions depicted in Figs. 15 and 16, despite the large difference in resolutions in each region between the two stretched-grid simulations.

\section{c. Tropical cyclones}

All three simulations produce too many LLTCs compared to IBTrACS (Table 6), with the c384 simulation having over 100 TCs per year, and the Taiwan simulation the least; this pattern is explained largely by the c384 simulation having the most LLTCs in the active western Pacific (WPac), and the Taiwan simulation the least. In the eastern Pacific (EPac), the c384 simulation again has the most LLTCs, but now the OKC simulation has the least; and in the North Atlantic (NAtl), the Taiwan simulation has the most and the OKC simulation has the least.

The worldwide number of TCs reaching $32.5 \mathrm{~m} \mathrm{~s}^{-1}$, including hurricanes and typhoons, is too large compared to IBTrACS (Table 6) in the uniform-resolution (a) NCEP Reanalysis

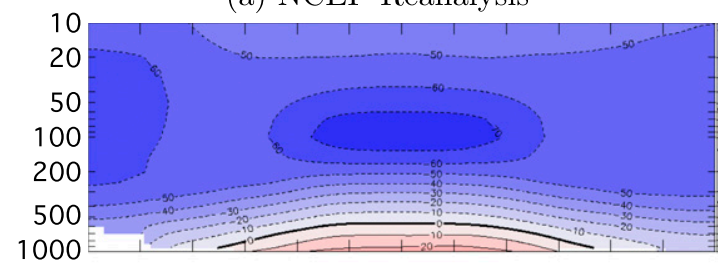

(c) c384r25 OKC (b) c384

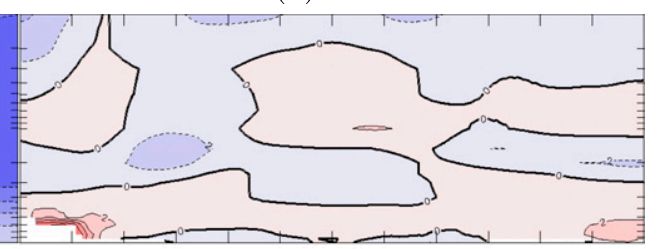

(d) c384r25 Taiwan

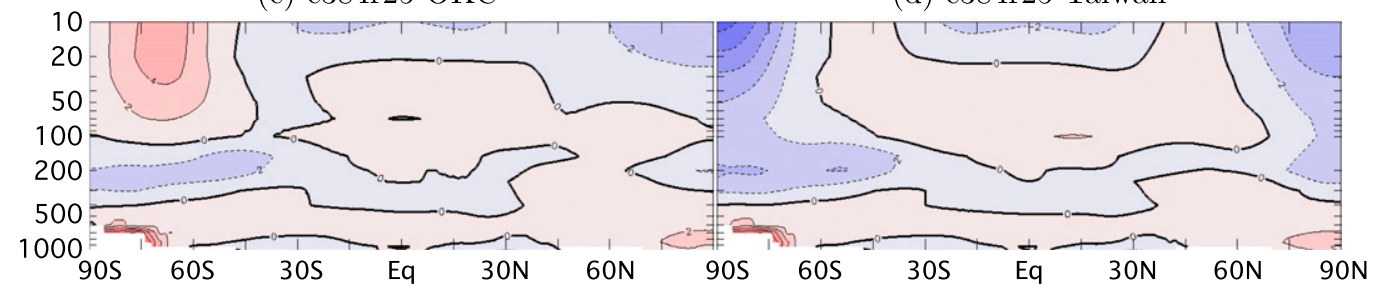

FIG. 13. As in Fig. 11, but for zonal-mean temperature. Contour interval is $10^{\circ} \mathrm{C}$ in (a) and $2^{\circ} \mathrm{C}$ in (b)-(d). 
(a) GPCP

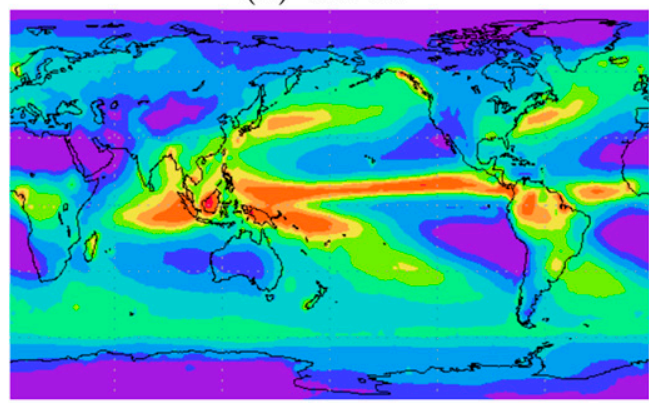

(c) $\mathrm{c} 384 \mathrm{r} 25 \mathrm{OKC}$

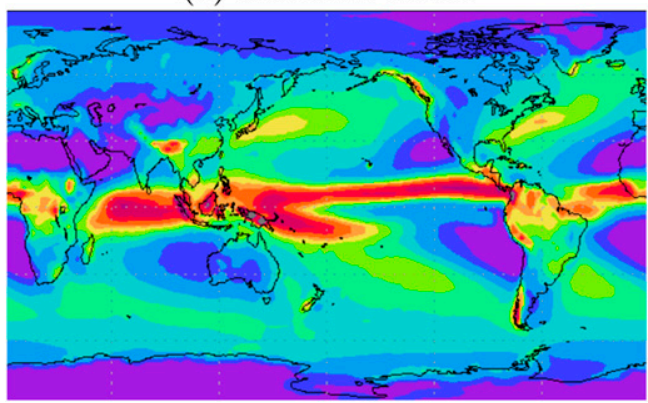

(b) c384

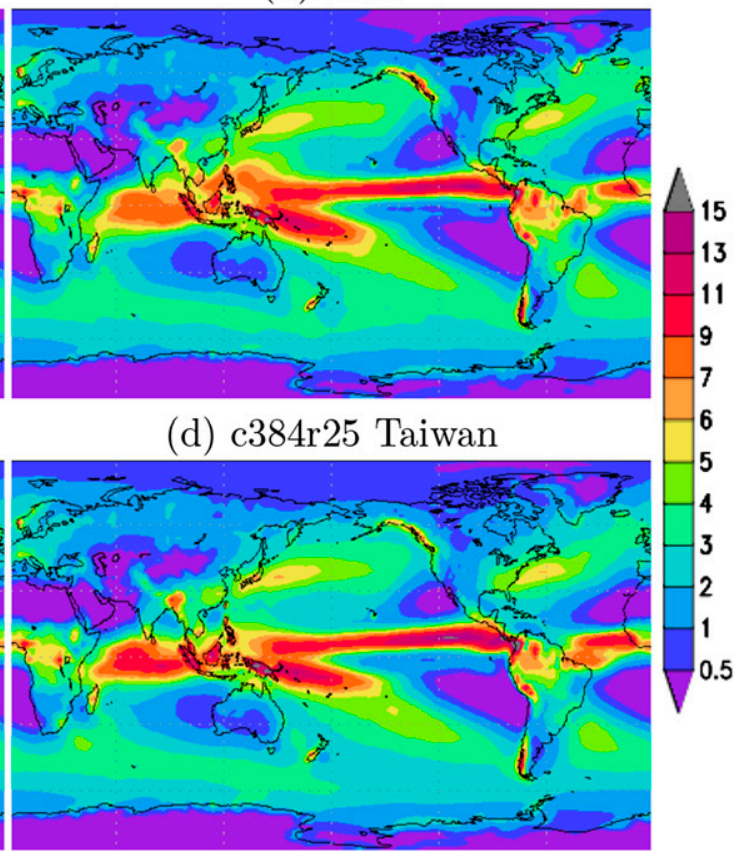

FIG. 14. Annual precipitation ( $\mathrm{mm} \mathrm{day}^{-1}$ ) in GPCP and HiRAM climate simulations: (a) GPCP, (b) c384, (c) c384r25 Oklahoma City, and (d) c384r25 Taiwan.

simulation and too low in the Taiwan stretched-grid simulation, with the OKC simulation having a minor high bias. The Taiwan simulation has the least, and the uniform-resolution simulation the most, in the western and eastern Pacific, and vice versa in the North Atlantic. Figure 19 demonstrates that the Taiwan simulation has too few typhoons throughout the western Pacific warm pool, the opposite of the uniform-resolution simulation, but both have too few cyclones in the South China Sea.
The Taiwan simulation does reduce the positive bias in cyclones in the north Indian Ocean and substantially reduces the positive bias around Australia compared to the uniform-resolution simulation. Also notable is that while the OKC simulation has a low bias in the eastern Pacific off of Mexico, it does not have the erroneously large number of hurricanes off of Central America seen in the other two simulations; both the low bias and high bias become more pronounced at increasingly low resolution.
TRMM Satellite
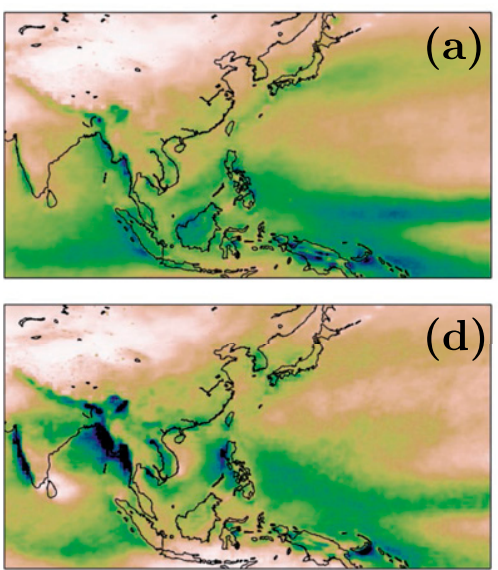

c384
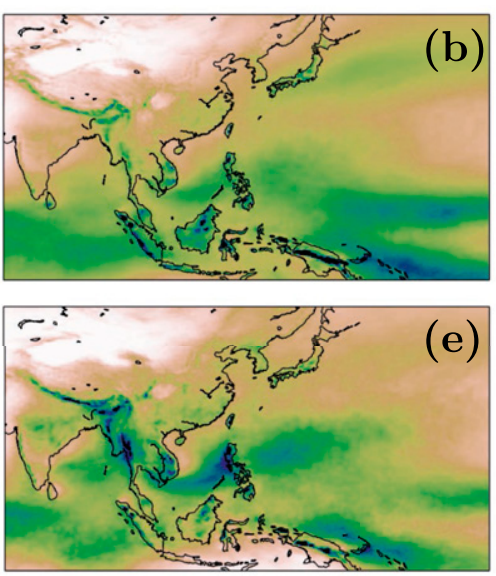

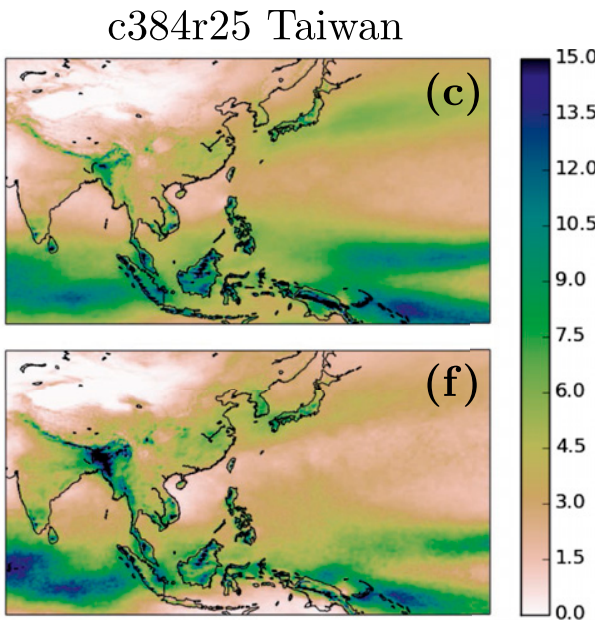

FIG. 15. Precipitation ( $\mathrm{mm} \mathrm{day}^{-1}$ ) for (top) annual and (bottom) monsoon-season (JJA) : (a),(d) TRMM v7; (b),(e) c384; and (c),(f) c384r25 Taiwan. The color scale is from 0 to $15.0 \mathrm{~mm} \mathrm{day}^{-1}$ in increments of $1.5 \mathrm{~mm} \mathrm{day}^{-1}$. 
TRMM Satellite
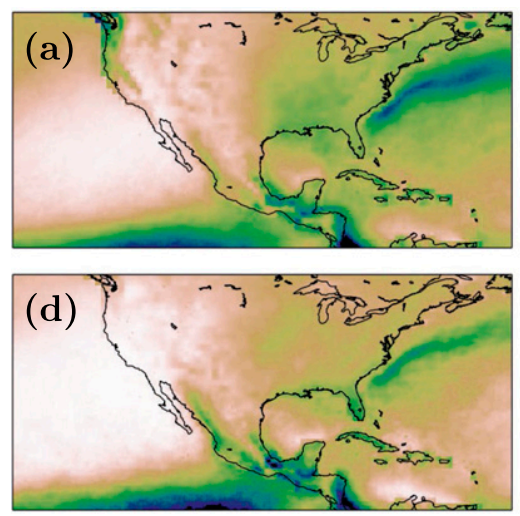

c384
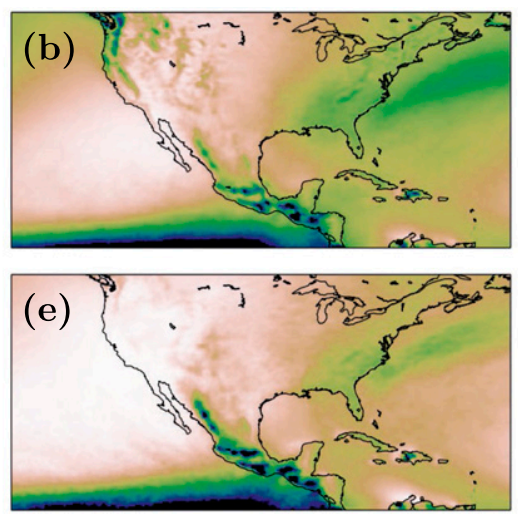

c384r 25 OKC
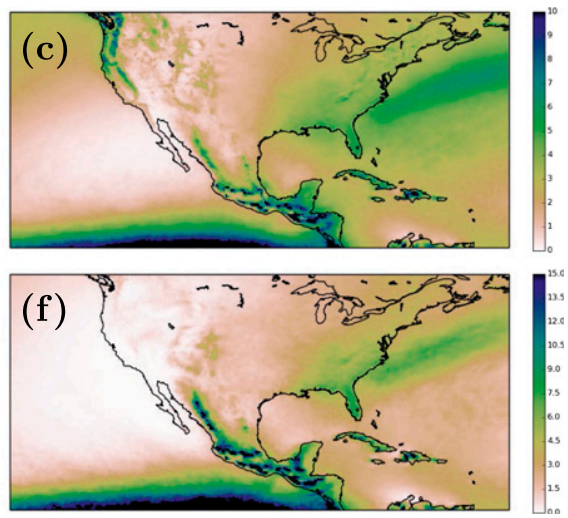

FIG. 16. Precipitation (mm day ${ }^{-1}$ ) for (top) annual and (bottom) warm-season (JJA): (a),(d) TRMM v7; (b),(e) c384; and (c),(f) c384r25 Oklahoma City. The color scale for (a)-(c) is from 0 to $10 \mathrm{~mm} \mathrm{day}^{-1}$ in increments of $1 \mathrm{~mm} \mathrm{day}^{-1}$ and for (d)-(f) is from 0 to $15.0 \mathrm{~mm} \mathrm{day}^{-1}$ in increments of $1.5 \mathrm{~mm} \mathrm{day}^{-1}$.

The Taiwan simulation did improve the interannual variability of western Pacific TCs and typhoons, improving the 1990-2008 correlation of both typhoons and LLTCs (Table 7). No improvement of interannual variability was seen in the eastern Pacific or North Atlantic in the OKC simulation. Note that only a single realization of each of the simulations is presented here, limiting the conclusions to be drawn from correlations of observed against modeled storm counts.

Biases in the number of hurricanes and typhoons are at least in part due to biases in the number of LLTCs. The worldwide fraction of LLTCs reaching $32.5 \mathrm{~m} \mathrm{~s}^{-1}$ is roughly $50 \%$ in all three simulations (Table 8 ), which is lower than observed. This is in part due to the tendency for all but the very highest-resolution models to produce too many tropical storm-strength storms and minimal hurricanes and typhoons. All three simulations produce too many weaker storms in both the western Pacific and North Atlantic (Fig. 20). The Taiwan stretched-grid simulation produces roughly the same number of very strong typhoons $\left(>55 \mathrm{~m} \mathrm{~s}^{-1}\right)$ as observed in the western Pacific, once a multiplicative factor of 1.05 is applied to adjust the 10-min Japanese Meteorological Agency winds to the 1-min winds used by other agencies and in other basins (dashed line in Fig. 20; Harper et al. 2010). The Taiwan stretched-grid simulation has fewer weak storms and more strong storms than the other simulations with lower resolutions over the western Pacific. Over the North Atlantic, the OKC stretched-grid simulation produces more strong hurricanes than the other simulations, albeit still fewer than observed. Indeed, in the North Atlantic and the eastern Pacific, the OKC simulation has the largest percentage of LLTCs becoming hurricanes out of all the simulations (Table 8). (a) PRISM

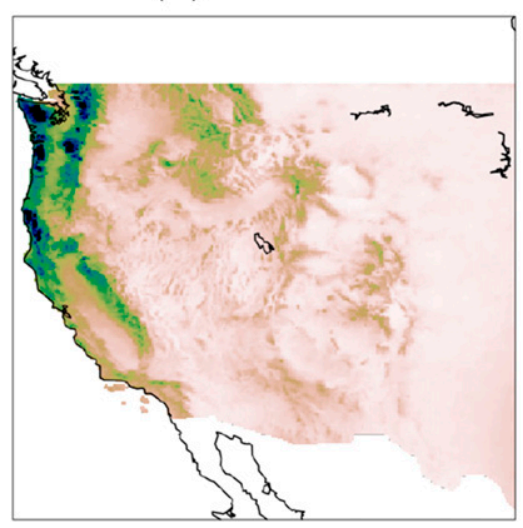

(b) c384

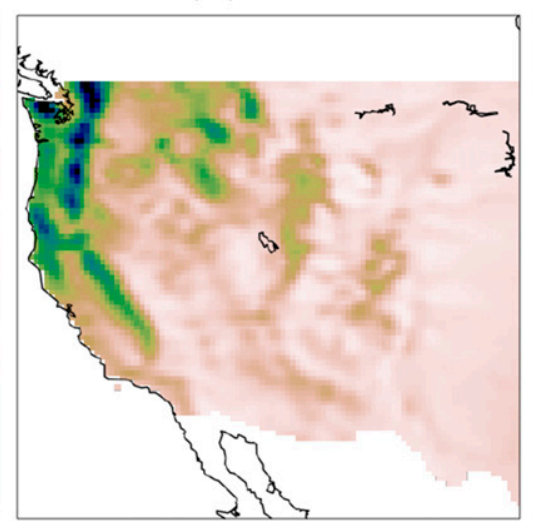

(c) $\mathrm{c} 384 \mathrm{r} 25 \mathrm{OKC}$

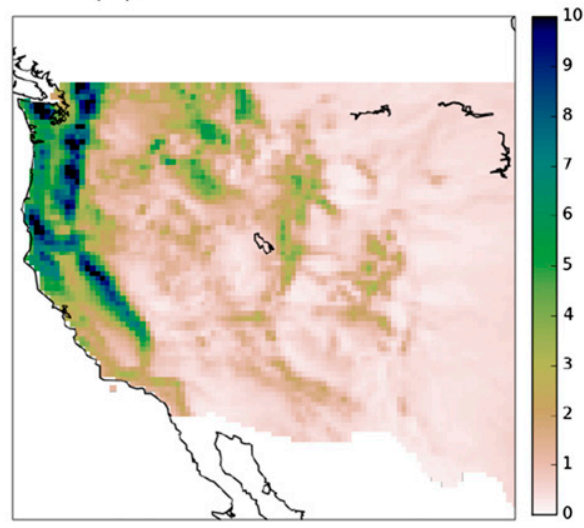

FIG. 17. Cold-season (DJF) western U.S. precipitation $\left(\mathrm{mm} \mathrm{day}^{-1}\right.$ ): (a) PRISM, (b) c384, and (c) c384r25 Oklahoma City. The color scale is from 0 to $10 \mathrm{~mm} \mathrm{day}^{-1}$ in increments of $1 \mathrm{~mm} \mathrm{day}^{-1}$. 


\section{Dates: 1980-2008 JJA \\ Observation: TRMM Dates: 1998-2012 (shifted 0 hours)}
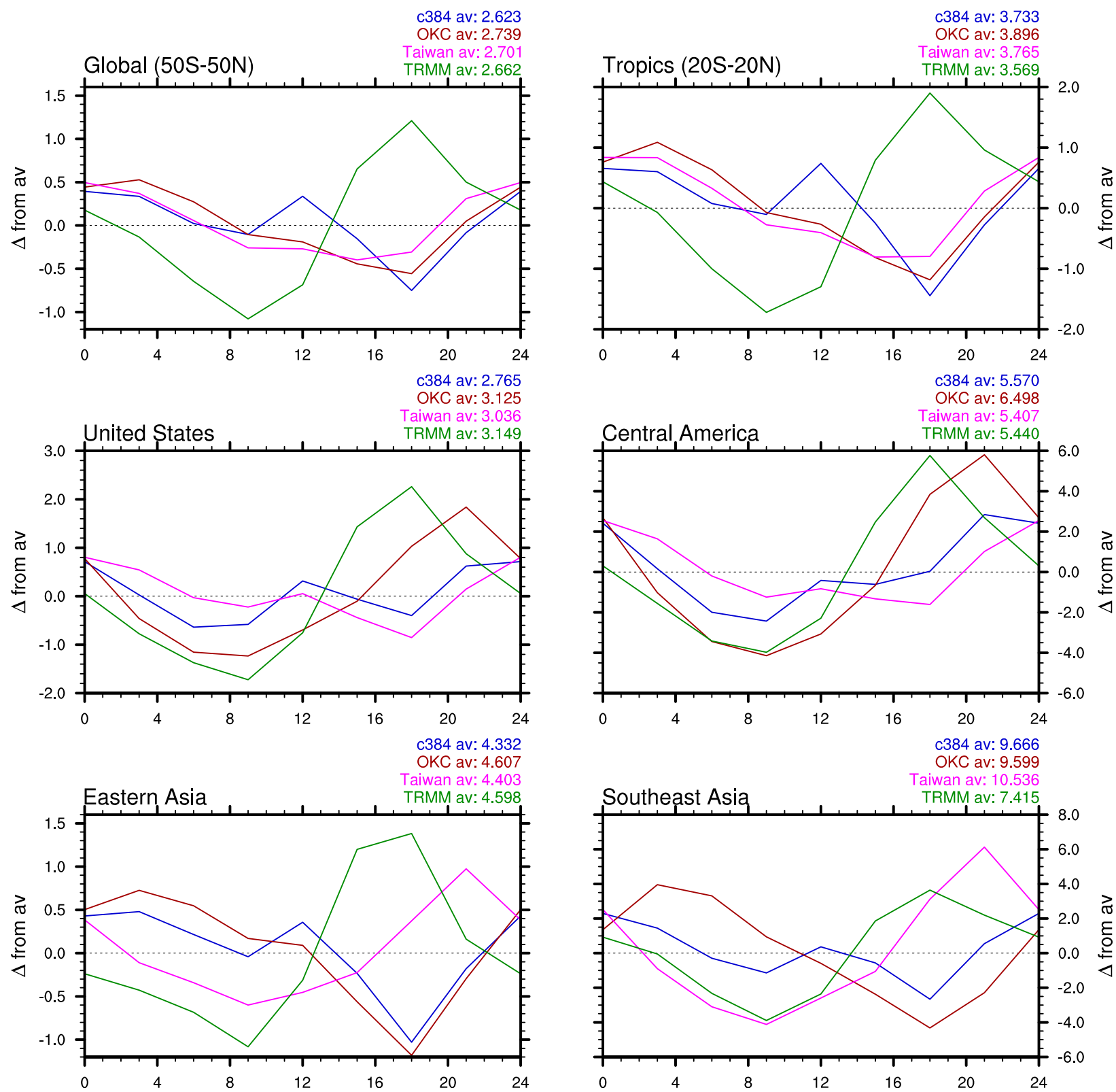

FIG. 18. The 1980-2008 JJA diurnal cycle over land for various regions. Panels depict deviation from daily-mean precipitation $\left(\mathrm{mm} \mathrm{day}^{-1}\right.$ ) as a function of local time (0000-2400): c384 (blue), c384r25 Oklahoma City (brown), and c384r25 Taiwan (pink). TRMM v7 (green curve) data is used for verification.

Furthermore, in the western Pacific the Taiwan stretchedgrid simulation has the largest percentage of LLTCs becoming typhoons. Both of these points confirm the conclusions from the aquaplanet simulations. The uniformresolution c384 simulation has the smallest percentage of North Atlantic and western Pacific LLTCs achieving hurricane strength, at least in part because the convective entrainment parameter is smaller than in the stretchedgrid simulations, which is known to decrease the ratio of hurricanes to LLTCs in HiRAM (Zhao et al. 2012). In the eastern Pacific, the percentage of LLTCs becoming hurricanes increases with improved resolution. 
TABLE 6. Number of LLTCs and hurricane-strength TCs per year, compared to 1990-2012 IBTrACS observations.

\begin{tabular}{|c|c|c|c|c|c|c|c|c|}
\hline & \multicolumn{4}{|c|}{ LLTCs } & \multicolumn{4}{|c|}{ Hurricane-strength TCs $\left(>32.5 \mathrm{~m} \mathrm{~s}^{-1}\right)$} \\
\hline & World & NAtl & $\mathrm{EPac}$ & WPac & World & NAtl & $\mathrm{EPac}$ & WPac \\
\hline c384 & 101.1 & 9.0 & 14.0 & 34.0 & 52.4 & 3.9 & 7.9 & 20.2 \\
\hline c384r25 OKC & 81.0 & 8.1 & 11.1 & 22.7 & 41.4 & 4.6 & 6.6 & 14.3 \\
\hline c384r25 Taiwan & 67.9 & 11.7 & 11.9 & 13.5 & 33.9 & 5.4 & 5.4 & 9.0 \\
\hline IBTrACS & 64.6 & 10.6 & 13.0 & 21.5 & 40.2 & 6.9 & 8.4 & 13.6 \\
\hline
\end{tabular}

\section{Conclusions}

Through a series of both idealized perpetual-September aquaplanet simulations and comprehensive climate simulations, we have demonstrated the ability of stretched grids in HiRAM to improve the simulated climate, with the largest improvements coming in the improved representation of finer-scale featuresorographic precipitation, the diurnal cycle of precipitation, and tropical cyclone intensity-over the region of enhanced resolution. We have also shown that despite the degradation of model resolution on the opposite side of the earth from the enhancedresolution region, the simulation fidelity of the large-scale climate is preserved compared to a uniformresolution simulation. Further, grid stretching has been shown to produce little in the way of numerical artifacts; instead, there is a gradual transition to a climate more like that of a higher-resolution simulation, as seen through the aquaplanet ITCZ position and in the increased fraction of tropical cyclones attaining hurricane strength, and the presence of major hurricanes and

(a) IBTrACS World TCs

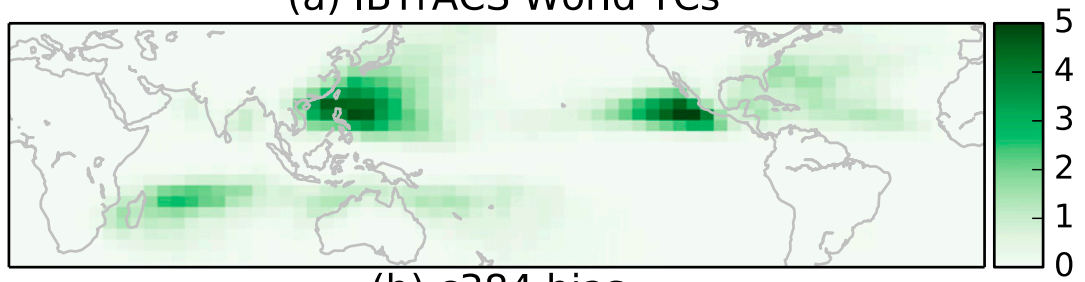

(b) c384 bias

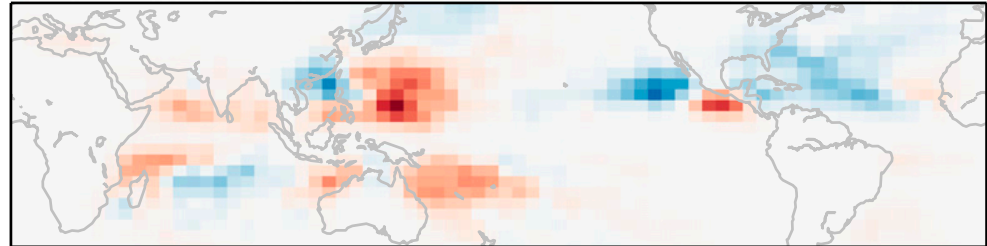

(c) c384r25 OKC bias

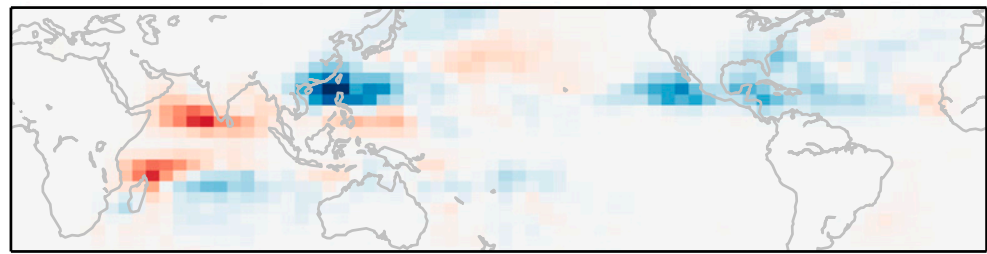

(d) c384r25 Taiwan bias

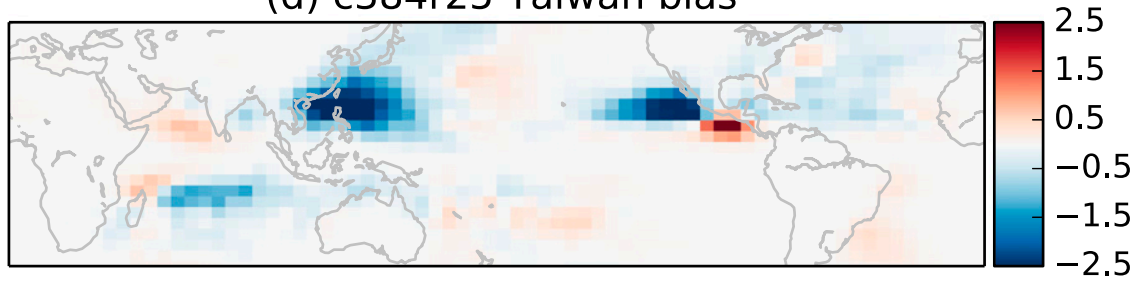

2.5

0.5

$-0.5$

$-2.5$

FIG. 19. (a) IBTrACS 1990-2012 storm density of hurricanes-typhoons (storm days per year). Biases in storm density (storm days per year, relative to IBTrACS) in (b) c384, (c) c384r25 Oklahoma City, and (d) c384r25 Taiwan. 
TABLE 7. Correlation of annual counts of LLTCs and hurricane-strength TCs with 1990-2012 IBTrACS observations.

\begin{tabular}{|c|c|c|c|c|c|c|}
\hline & \multicolumn{3}{|c|}{ LLTCs } & \multicolumn{3}{|c|}{ Hurricane-strength TCs $\left(>32.5 \mathrm{~m} \mathrm{~s}^{-1}\right)$} \\
\hline & NAtl & $\mathrm{EPac}$ & WPac & NAtl & EPac & WPac \\
\hline c384 & 0.55 & 0.60 & 0.41 & 0.55 & 0.41 & 0.32 \\
\hline c384r25 OKC & 0.45 & -0.02 & 0.48 & 0.49 & 0.33 & 0.30 \\
\hline c384r25 Taiwan & 0.46 & -0.05 & 0.73 & 0.50 & 0.09 & 0.47 \\
\hline
\end{tabular}

supertyphoons not apparent at lower resolutions. Improvements due to the stretched grid are mostly due to resolution, since there was very little parameterization tuning to the stretched-grid simulations, and the parameterizations used are not explicitly scale-aware.

Aquaplanet simulations provide an idealized environment to demonstrate the gradual variation in precipitation and tropical cyclones with the gradual variations in gridcell width. Indeed, in the stretched-grid aquaplanet simulation with gridcell widths ranging from 12 to $130 \mathrm{~km}$, the latitudinal position and intensity of the ITCZ, as well as the number of TCs and the fraction of which achieved hurricane $\left(32.5 \mathrm{~m} \mathrm{~s}^{-1}\right)$ strength, closely matched those in corresponding uniform-resolution simulations. The stretched-grid aquaplanet simulation was largely free of grid artifacts except for an artificial circulation resembling a Walker circulation in the tropics.

There was no general degradation of the large-scale climate in the stretched-grid comprehensive climate simulations despite the range of grid scales, the lowresolution region, or the compromise parameterization tuning. In fact, for dynamical fields such as the zonalmean winds and mean Northern Hemisphere pressure, errors were reduced in the stretched-grid simulations compared to a $25-\mathrm{km}$ uniform-resolution simulation. Enhanced resolutions as high as $10 \mathrm{~km}$ from the stretched grid also locally improved orographic precipitation, although improvements in nonorographic precipitation biases were rather more equivocal. Alleviation of these biases may require advances in physical parameterizations, which lies beyond the scope of this paper.

Over land, the diurnal cycle in the 25-km uniformresolution simulation tended to erroneously peak near local noon and at midnight, and did little better than the 60-70-km low-resolution regions of the stretched-grid simulations; but when the stretched grid locally increased the resolution to $10 \mathrm{~km}$, the diurnal cycle was nearly always improved, with the peak now occurring within $3 \mathrm{~h}$ of the typical early evening observed peak. As expected, enhanced resolution was shown to increase the number of the most intense tropical cyclones, particularly in the North Atlantic. The stretched grid was also able to alleviate positive biases in many basins through enhanced resolution. No grid artifacts due to the stretched grid were apparent in these climate simulations.
Two results from the aquaplanet simulations help to illuminate results seen in the comprehensive climate simulations. The highest-resolution regions of the stretched-grid climate simulations have the fewest TCs compared to the coarser-resolution simulations of the same region, and thus may not have as many hurricanestyphoons as one of the coarser simulations, but they always have the largest percentage of TCs that attain hurricane/typhoon strength. The same result occurred in the aquaplanet simulations: at higher resolutions fewer overall TCs formed, attributable to the ITCZ shifting to lower latitudes and thereby providing a less favorable environment for TC genesis. However, since the higher resolution allows the model to better resolve intense cyclones, more of the storms that did form were able to attain hurricane strength.

Second, the ITCZ in the low-resolution region of the stretched-grid aquaplanet simulation was better defined and more intense, more like that of the higher-resolution regions of the stretched-grid simulation, than that of the uniform-resolution $110-\mathrm{km}$ simulation, implying that the better-resolved regions may have a positive effect upon the lower-resolution regions of the stretched grid. This in turn may be similar to the effect seen in the stretched-grid climate simulations, in which SLP and zonal-mean winds were globally improved despite much of the domain having a degraded resolution compared to a uniformresolution simulation.

We have demonstrated the utility of the stretched grid to both locally and potentially globally improve the fidelity of a simulated climate, both with respect to mean climate and to variability such as TCs. Further work will involve even higher-resolution simulations, such as with a combination of grid stretching and grid nesting, nonhydrostatic climate simulations, and time-slice simulations forced with late twenty-first-century SSTs.

TABLE 8. Percentage of LLTCs reaching hurricane/typhoon strength compared to 1990-2012 IBTrACS observations.

\begin{tabular}{lcccc}
\hline \hline & World (\%) & Natl (\%) & EPac (\%) & WPac (\%) \\
\hline c384 & 51.8 & 43.3 & 56.4 & 59.4 \\
c384r25 OKC & 51.1 & 56.8 & 59.4 & 63.0 \\
c384r25 Taiwan & 49.9 & 46.2 & 45.4 & 66.7 \\
IBTrACS & 62.2 & 79.2 & 64.6 & 63.3 \\
\hline
\end{tabular}



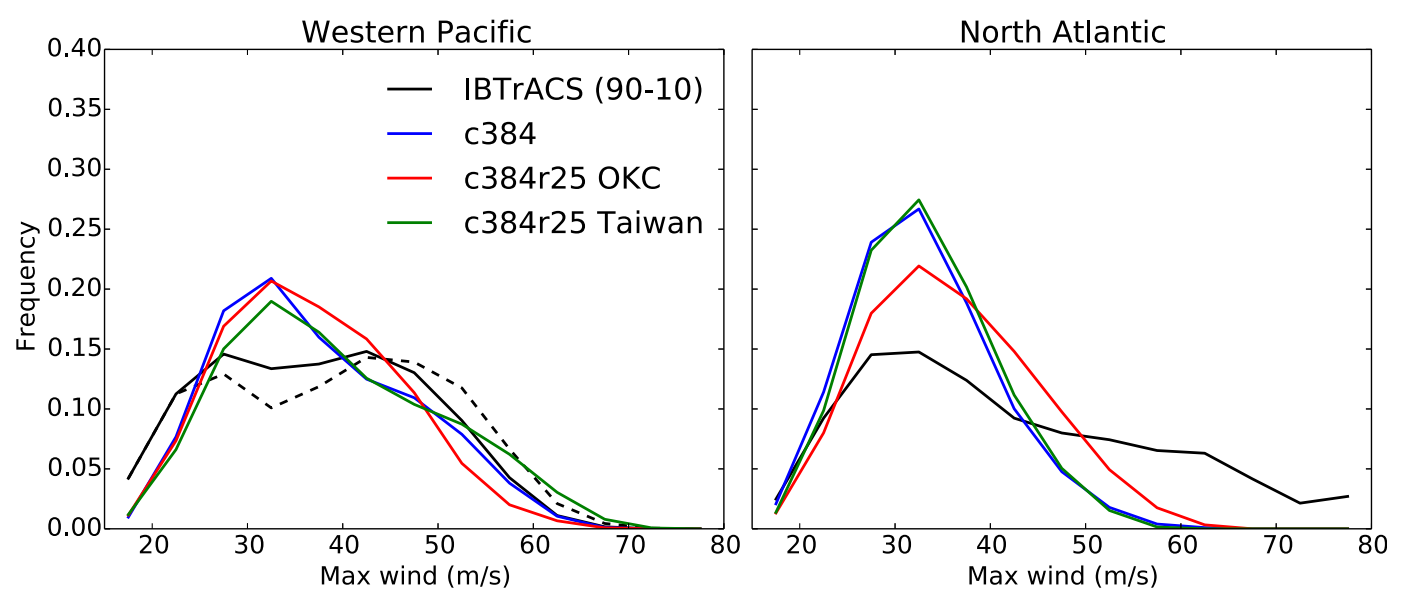

FIG. 20. Probability distribution of lifetime-maximum intensity of LLTCs in (left) the western Pacific and (right) North Atlantic. Dashed black line represents the adjustment (Harper et al. 2010) over the western Pacific using the Japan Meteorological Agency (JMA) 10-min winds to the 1-min winds used in the North Atlantic.

Acknowledgments. Zhi Liang, Rusty Benson, and Sergei Malychev provided indispensable technical assistance. Aquaplanet HiRAM was designed by Ming Zhao and Bruce Wyman, and the simulations were originally constructed by Chris Kerr. Sarah Kapnick provided valuable discussion of TRMM biases. IBTrACS data were sorted and quality-controlled by Jan-Huey Chen. Diurnal cycle plots were made using a script designed by Bruce Wyman. Discussions with Tim Marchok, Ming Zhao, JanHuey Chen, Baoqiang Xiang, and Song-Miao Fan were very helpful in developing and testing the tracker. Tom Knutson and Tim Marchok provided valuable internal reviews. Comments from three anonymous reviewers are greatly appreciated.

\section{APPENDIX}

\section{Description of the Cyclone Tracker}

Tracking is performed on 6-hourly instantaneous SLP, $10-\mathrm{m}$ winds, and upper-tropospheric $(300-500 \mathrm{hPa})$ temperature. First, storm centers are found, identified by minima in the SLP field of depression at least $2 \mathrm{hPa}$ compared to their surroundings. This is done by finding a minimum, no greater than $1013 \mathrm{hPa}$, in an SLP field that has been smoothed with a second-order filter, and then searching for a closed contour $2 \mathrm{hPa}$ greater than the minimum not encircling any deeper minima. Closed contours are identified by using a "flood fill" algorithm in which all adjacent cells with pressures less than the value of the contour are found, creating a region bounded by that contour. This flood fill method is easily and efficiently implemented using a recursive algorithm and does not require the contour to be circular. Further closed contours are used to identify the region associated with this cyclone, in which $10-\mathrm{m}$ wind speed and 300-500-hPa temperature maxima are searched for; if the maximum in 300-500-hPa temperature is encircled by a $2^{\circ} \mathrm{C}$ contour and is no more than $500 \mathrm{~km}$ from the storm center, then the storm is considered to have a warm core. To filter out weak or disorganized systems, the identified region must also have a maximum $850-\mathrm{hPa}$ cyclonic vorticity of at least $1.5 \times 10^{-4} \mathrm{~s}^{-1}$ (negative in the Southern Hemisphere). The location of the center is fine-tuned by fitting a biquadratic to the SLP field and placing the center at its minimum.

Storms identified at successive times can be connected into a track if they satisfy proximity criteria. Storms are assumed to move between data times along a path extrapolated from their two previous times, or using $500-\mathrm{hPa}$ winds if there is only one earlier time; if an extrapolated storm center lies within $750 \mathrm{~km}$ of an identified storm center at the current time they are connected into a track. Identification proceeds in order of lowest to highest central SLP for both current and previous storms; doing so avoids problems in which a strong storm at the previous time is associated with a nearby weak disturbance at the current time while the correct current-time storm is counted as a new storm track.

The criteria of Chen and Lin $(2011,2013)$ are used to identify LLTCs. The total lifetime of a storm track must be at least $72 \mathrm{~h}$ (regardless of maximum wind speed), must have a warm core and maximum winds of at least tropical-storm strength $\left(17.5 \mathrm{~m} \mathrm{~s}^{-1}\right)$ for at least 36 consecutive hours, and must have a warm core for a cumulative total of at least $48 \mathrm{~h}$ (not necessarily consecutive). A hurricane-strength storm is any storm satisfying the 72-h lifetime requirement and having hurricane-strength winds at any time, so long as the storm center is 
equatorward of $40^{\circ}$ latitude. A hurricane-strength storm need not satisfy the other lifetime requirements, including the warm-core criterion.

When a minimum central pressure criterion is used instead of a maximum wind speed criterion, the longevity and threshold criteria are the same except that the tropical storm wind speed threshold is replaced with the requirement that the minimum SLP is less than $1000 \mathrm{hPa}$, and that the hurricane wind speed threshold is replaced with a $980-\mathrm{hPa}$ minimum SLP threshold.

\section{REFERENCES}

Adler, R. F., and Coauthors, 2003: The version-2 Global Precipitation Climatology Project (GPCP) monthly precipitation analysis (1979-present). J. Hydrometeor., 4, 1147-1167, doi:10.1175/1525-7541(2003)004<1147:TVGPCP > 2.0.CO;2.

Anderson, J. L., and Coauthors, 2004: The new GFDL global atmosphere and land model AM2-LM2: Evaluation with prescribed SST simulations. J. Climate, 17, 4641-4673, doi:10.1175/JCLI-3223.1.

Ballinger, A. P., T. M. Merlis, I. M. Held, and M. Zhao, 2015: The sensitivity of tropical cyclone activity to off-equatorial thermal forcing in aquaplanet simulations. J. Atmos. Sci., 72, 22862302, doi:10.1175/JAS-D-14-0284.1.

Bender, M. A., T. R. Knutson, R. E. Tuleya, J. J. Sirutis, G. A. Vecchi, S. T. Garner, and I. M. Held, 2010: Modeled impact of anthropogenic warming on the frequency of intense Atlantic hurricanes. Science, 327, 454-458, doi:10.1126/ science. 1180568 .

Blackburn, M., and Coauthors, 2013: The Aqua-Planet Experiment (APE): CONTROL SST simulation. J. Meteor. Soc. Japan, 91A, 17-56, doi:10.2151/jmsj.2013-A02.

Bretherton, C. S., J. R. McCaa, and H. Grenier, 2004: A new parameterization for shallow cumulus convection and its application to marine subtropical cloud-topped boundary layers. Part I: Description and 1D results. Mon. Wea. Rev., 132, 864-882, doi:10.1175/1520-0493(2004)132<0864:ANPFSC > 2.0.CO;2.

Chen, J.-H., and S.-J. Lin, 2011: The remarkable predictability of inter-annual variability of Atlantic hurricanes during the past decade. Geophys. Res. Lett., 38, L11804, doi:10.1029/2011GL047629.

$\ldots$, and _ 2013: Seasonal predictions of tropical cyclones using a $25-\mathrm{km}$ resolution general circulation model. J. Climate, 26, 380-398, doi:10.1175/JCLI-D-12-00061.1.

Colella, P., and P. R. Woodward, 1984: The piecewise parabolic method (PPM) for gas-dynamical simulations. J. Comput Phys., 54, 174-201, doi:10.1016/0021-9991(84)90143-8.

Courtier, P., and J.-F. Geleyn, 1988: A global numerical weather prediction model with variable resolution: Application to the shallow-water equations. Quart. J. Roy. Meteor. Soc., 114, 1321-1346, doi:10.1002/qj.49711448309.

Daly, C., M. Halbleib, J. I. Smith, W. P. Gibson, M. K. Doggett, G. H. Taylor, J. Curtis, and P. P. Pasteris, 2008: Physiographically sensitive mapping of climatological temperature and precipitation across the conterminous United States. Int. J. Climatol., 28, 2031-2064, doi:10.1002/joc.1688.

Déqué, M., and J. Piedelievre, 1995: High resolution climate simulation over Europe. Climate Dyn., 11, 321-339, doi:10.1007/ BF00215735.

Fox-Rabinovitz, M., J. Côté, B. Dugas, M. Déqué, and J. L. McGregor, 2006: Variable resolution general circulation models:
Stretched-grid model intercomparison project (SGMIP). J. Geophys. Res, 111, D16104, doi:10.1029/2005JD006520.

Gall, J. S., I. Ginis, S.-J. Lin, T. P. Marchok, and J.-H. Chen, 2011: Experimental tropical cyclone prediction using the GFDL 25-km-resolution global atmospheric model. Wea. Forecasting, 26, 1008-1019, doi:10.1175/WAF-D-10-05015.1.

Gopalan, K., N.-Y. Wang, R. Ferraro, and C. Liu, 2010: Status of the TRMM 2A12 land precipitation algorithm. J. Atmos. Oceanic Technol., 27, 1343-1354, doi:10.1175/2010JTECHA1454.1.

Gray, W. M., 1968: Global view of the origin of tropical disturbances and storms. Mon. Wea. Rev., 96, 669-700, doi:10.1175/ 1520-0493(1968)096<0669:GVOTOO>2.0.CO;2.

Halperin, D. J., H. E. Fuelberg, R. E. Hart, J. H. Cossuth, P. Sura, and R. J. Pasch, 2013: An evaluation of tropical cyclone genesis forecasts from global numerical models. Wea. Forecasting, 28, 1423-1445, doi:10.1175/WAF-D-13-00008.1.

Harper, B., J. Kepert, and J. Ginger, 2010: Guidelines for converting between various wind averaging periods in tropical cyclone conditions. WMO/TD-1555, $52 \mathrm{pp}$.

Harris, L. M., and S.-J. Lin, 2013: A two-way nested global-regional dynamical core on the cubed-sphere grid. Mon. Wea. Rev., 141, 283-306, doi:10.1175/MWR-D-11-00201.1.

, and - 2014: Global-to-regional nested-grid climate simulations in the GFDL High Resolution Atmosphere Model. J. Climate, 27, 4890-4910, doi:10.1175/JCLI-D-13-00596.1.

Held, I. M., M. Zhao, and B. Wyman, 2007: Dynamic radiativeconvective equilibria using GCM column physics. J. Atmos. Sci., 64, 228-238, doi:10.1175/JAS3825.11.

Huffman, G. J., and Coauthors, 2007: The TRMM Multisatellite Precipitation Analysis (TMPA): Quasi-global, multiyear, combined-sensor precipitation estimates at fine scales. J. Hydrometeor., 8, 38-55, doi:10.1175/JHM560.1.

Lin, S.-J., 1997: A finite-volume integration method for computing pressure gradient force in general vertical coordinates. Quart. J. Roy. Meteor. Soc., 123, 1749-1762, doi:10.1002/ qj. 49712354214.

_ 2004: A "vertically Lagrangian" finite-volume dynamical core for global models. Mon. Wea. Rev., 132, 2293-2307, doi:10.1175/1520-0493(2004)132<2293:AVLFDC>2.0.CO;2.

__ , and R. Rood, 1996: Multidimensional flux-form semiLagrangian transport schemes. Mon. Wea. Rev., 124, 2046-2070, doi:10.1175/1520-0493(1996)124<2046:MFFSLT>2.0.CO;2.

, and —, 1997: An explicit flux-form semi-Lagrangian shallow-water model on the sphere. Quart. J. Roy. Meteor. Soc., 123, 2477-2498, doi:10.1002/qj.49712354416.

Lin, Y.-L., R. D. Farley, and H. D. Orville, 1983: Bulk parameterization of the snow field in a cloud model. J. Climate Appl. Meteor., 22, 1065-1092, doi:10.1175/1520-0450(1983)022<1065: $\mathrm{BPOTSF}>2.0 . \mathrm{CO} ; 2$.

Lorant, V., and J.-F. Royer, 2001: Sensitivity of equatorial convection to horizontal resolution in aquaplanet simulations with a variable-resolution GCM. Mon. Wea. Rev., 129, 2730-2745, doi:10.1175/1520-0493(2001)129<2730:SOECTH >2.0.CO;2.

McGregor, J. L., 2005: C-CAM: Geometric aspects and dynamical formulation. CSIRO Atmospheric Research Tech. Paper 70, 43 pp. [Available online at https://publications. csiro.au/rpr/pub?list=BRO\&pid= procite $:$ eb33ff33-c6d6-47798b82-867b2ca6e112.]

- 2015: Recent developments in variable-resolution global climate modelling. Climatic Change, 129, 369-380, doi:10.1007/ s10584-013-0866-5.

, and M. R. Dix, 2008: An updated description of the conformalcubic atmospheric model. High Resolution Numerical Modelling 
of the Atmosphere and Ocean, K. Hamilton and W. Ohfuchi, Eds., Springer, 51-75.

Merlis, T. M., M. Zhao, and I. M. Held, 2013: The sensitivity of hurricane frequency to ITCZ changes and radiatively forced warming in aquaplanet simulations. Geophys. Res. Lett., 40, 4109-4114, doi:10.1002/grl.50680.

Milly, P., and Coauthors, 2014: An enhanced model of land water and energy for global hydrologic and Earth-system studies. J. Hydrometeor., 15, 1739-1761, doi:10.1175/JHM-D-13-0162.1.

Putman, W. M., and S.-J. Lin, 2007: Finite-volume transport on various cubed-sphere grids. J. Comput. Phys., 227, 55-78, doi:10.1016/j.jcp.2007.07.022.

Rauscher, S. A., T. D. Ringler, W. C. Skamarock, and A. A. Mirin, 2013: Exploring a global multiresolution modeling approach using aquaplanet simulations. J. Climate, 26, 2432-2452, doi:10.1175/JCLI-D-12-00154.1.

Rayner, N., D. Parker, E. Horton, C. Folland, L. Alexander, D. Rowell, E. Kent, and A. Kaplan, 2003: Global analyses of sea surface temperature, sea ice, and night marine air temperature since the late nineteenth century. J. Geophys. Res., 108, 4407, doi:10.1029/2002JD002670.

Satoh, M., and Coauthors, 2014: The non-hydrostatic icosahedral atmospheric model: description and development. Prog. Earth Planet. Sci., 1, 1-32, doi:10.1186/s40645-014-0018-1.

Schmidt, F., 1977: Variable fine mesh in spectral global models. Beitr. Phys. Atmos., 50, 211-217.

Simpson, J. J., J. S. Berg, C. J. Koblinsky, G. L. Hufford, and B. Beckley, 2001: The NVAP global water vapor data set: Independent cross-comparison and multiyear variability. Remote Sens. Environ., 76, 112-129, doi:10.1016/S0034-4257(00)00199-1.

Stern, W., and R. Pierrehumbert, 1988: The impact of an orographic gravity wave drag parameterization on extended range predictions with a GCM. Eighth Conf. on Numerical Weather Prediction, Baltimore, MD, Amer. Meteor. Soc., 745-750. [Available online at http://www.gfdl.noaa.gov/cms-filesystemaction/user_files/lmh/sternpierrehumbert.pdf.]

Thuburn, J., T. D. Ringler, W. C. Skamarock, and J. B. Klemp, 2009: Numerical representation of geostrophic modes on arbitrarily structured C-grids. J. Comput. Phys., 228, 8321-8335, doi:10.1016/j.jcp.2009.08.006.

Tomita, H., 2008: A stretched icosahedral grid by a new grid transformation. J. Meteor. Soc. Japan, 86A, 107-119, doi:10.2151/ jmsj.86A.107.

- H. Miura, S. Iga, T. Nasuno, and M. Satoh, 2005: A global cloud-resolving simulation: Preliminary results from an aqua planet experiment. Geophys. Res. Lett., 32, L08805, doi:10.1029/ 2005 GL022459.
Uchida, J., M. Mori, H. Nakamura, M. Satoh, K. Suzuki, and T. Nakajima, 2016: Error and energy budget analysis of a nonhydrostatic stretched-grid global atmospheric model. Mon. Wea. Rev., 144, 1423-1447, doi:10.1175/MWR-D-15-0271.1.

Walko, R., D. Medvigy, and R. Avissar, 2010: Toward a unified representation of atmospheric convection in variable resolution climate models. 2010 AGU Fall Meeting, San Francisco, CA, Amer. Geophys. Union, Abstract A24D-07.

Walsh, K., M. Fiorino, C. Landsea, and K. McInnes, 2007: Objectively determined resolution-dependent threshold criteria for the detection of tropical cyclones in climate models and reanalyses. J. Climate, 20, 2307-2314, doi:10.1175/ JCLI4074.1.

Wang, J.-J., R. F. Adler, G. J. Huffman, and D. Bolvin, 2014: An updated TRMM composite climatology of tropical rainfall and its validation. J. Climate, 27, 273-284, doi:10.1175/ JCLI-D-13-00331.1.

Williamson, D. L., 2008: Equivalent finite volume and Eulerian spectral transform horizontal resolutions established from aqua-planet simulations. Tellus, 60A, 839-847, doi:10.1111/ j.1600-0870.2008.00340.x.

_ - and Coauthors, 2013: The Aqua-Planet Experiment (APE): Response to changed meridional SST profile. J. Meteor. Soc. Japan, 91A, 57-89, doi:10.2151/jmsj.2013-A03.

Xiang, B., and Coauthors, 2015: Beyond weather time-scale prediction for Hurricane Sandy and Super Typhoon Haiyan in a global climate model. Mon. Wea. Rev., 143, 524-535, doi:10.1175/ MWR-D-14-00227.1.

Zarzycki, C. M., C. Jablonowski, D. R. Thatcher, and M. A. Taylor, 2015: Effects of localized grid refinement on the general circulation and climatology in the Community Atmosphere Model. J. Climate, 28, 2777-2803, doi:10.1175/JCLI-D-14-00599.1.

Zhao, M., and I. M. Held, 2010: An analysis of the effect of global warming on the intensity of Atlantic hurricanes using a GCM with statistical refinement. J. Climate, 23, 6382-6393, doi:10.1175/ 2010JCLI3837.1.

,,-- S.-J. Lin, and G. A. Vecchi, 2009: Simulations of global hurricane climatology, interannual variability, and response to global warming using a 50-km resolution GCM. J. Climate, 22 6653-6678, doi:10.1175/2009JCLI3049.1.

,$- \ldots$, and —_ 2012: Some counterintuitive dependencies of tropical cyclone frequency on parameters in a GCM. J. Atmos. Sci., 69, 2272-2283, doi:10.1175/JAS-D-11-0238.1.

Zhou, W., I. M. Held, and S. T. Garner, 2014: Parameter study of tropical cyclones in rotating radiative-convective equilibrium with column physics and resolution of a $25-\mathrm{km}$ GCM. J. Atmos. Sci., 71, 1058-1069, doi:10.1175/JAS-D-13-0190.1. 